\title{
Canonical maps near separatrix in Hamiltonian systems
}

\author{
S. S. Abdullaev \\ Institut für Plasmaphysik, Forschungszentrum Jülich GmbH, EURATOM Association, Trilateral Euregio Cluster, \\ D-52425 Jülich, Germany
}

(Received 24 March 2004; published 7 October 2004)

\begin{abstract}
A systematic and rigorous method to construct symplectic maps near separatrix of generic Hamiltonian systems subjected to time-periodic perturbations is developed. It is based on the method of canonical transformation of variables to construct Hamiltonian maps [S. S. Abdullaev, J. Phys. A 35, 2811 (2002)]. Using canonical transformation of variables and the first-order approximation for the generating function, the general form of mapping in terms of time and energy variables is obtained. Different limiting cases of the mapping are considered. The method is illustrated for simple Hamiltonian systems with one and a large number of saddle points. It is also applied to derive mappings for the periodic-driven Morse oscillator describing the process of stochastic excitation and dissociation of diatomic molecules. The so-called canonical Kepler map is derived for the one-dimensional hydrogen atom in a microwave field.
\end{abstract}

DOI: 10.1103/PhysRevE.70.046202

PACS number(s): 05.45.Ac, 45.05.+x, 45.10.Hj, 45.20.Jj

\section{INTRODUCTION}

Separatrices are curves in the phase space of dynamical systems connecting their saddle fixed points and separating different types of motion. A motion near separatrices of dynamical systems, especially Hamiltonian systems, has fundamental generic features. As was first shown by Poincaré [1], any small time-periodic perturbation splits separatrices corresponding to stable and unstable manifolds, which leads to the onset of chaotic motion due to the exponential divergence of orbits with close initial conditions [2,3]. This phenomenon creates the zone of phase space, i.e., a stochastic layer in the neighborhood of unperturbed separatrices where the motion of the system is chaotic.

The study of the structure of chaotic motion in the stochastic layer and transport associated with this motion by simple numerical integrations of the equations of motion requires long computational times. To study a motion near the separatrix, an iterative mapping, known as the separatrix (or whisker) mapping, has been proposed (see Refs. [3-5]) and its different modifications have been widely used in various problems of physics and astronomy. Particularly, in Refs. [6-12], the separatrix mapping has been applied to study a chaotic transport of passive particles in structured fluids, and in Refs. [13-16] to study magnetic-field lines in tokamaks. A particle-wave interaction in plasmas has been studied using the separatrix mapping in Refs. [5,17,18]. In Refs. [19-21], the separatrix map has been used to study the dynamics of asteroids in the Solar System and the rotational motion of a satellite. The so-called Kepler map proposed to study the classical ionization of hydrogen atoms in a microwave field [22-27] and the motion of comets in the Solar System [28-31] belongs also to the class of separatrix mappings.

References [32-34] were devoted to the rigorous derivation of the separatrix mapping. Particularly, a multidimensional analog of the separatrix mapping has been constructed in Ref. [34]. The different forms of the separatrix mapping or its generalizations have been discussed in Refs. [14,15,35-40]. Separatrix mappings played an instrumental role in the establishment of the rescaling invariant properties of Hamiltonian systems near saddle points and the chaotic transport in the stochastic layer (see Refs. [41-47]).

One of the main shortcomings of the separatrix mapping is that it is not a canonical mapping since, as was noticed by [17], the mapping variables-time and energy - are defined at different sections of the phase space. Therefore, it does not coincide with the Poincaré map. In principle, it makes it difficult to compare the phase-space structure of the system with that of the separatrix mapping. Therefore, the properties of separatrix mapping may not coincide with the properties of the original system. As was shown in Ref. [14], the rescaling invariance property of Hamiltonian systems near hyperbolic saddle points cannot be directly recovered from the conventional separatrix mapping.

We should also note an inconsistency which appears in the quantization problem of classical systems using noncanonical mappings. For instance, in several works (see Ref. [26]), attempts have been made to quantize the conventional (noncanonical) Kepler map to study quantum effects in the process of ionization of highly excited hydrogen atoms in a microwave. However, such a procedure of quantization of the Kepler map by presenting energy and time variables as a canonical pair of operators is not consistent with the fundamental principles of quantum mechanics, since the energy and time variables in this map are not canonically conjugated.

The conventional method to derive separatrix mappings is mainly based on the calculations of the increments of time and energy variables over phase rotation in phase space (see, for instance, Ref. [3]). This method does not allow us to directly obtain canonical separatrix mappings. On the other hand, this method does not allow us to estimate the accuracy of the separatrix mapping.

There were several studies to construct canonical separatrix mappings with variables defined at the same sections of phase space. Particularly, in $[14,15]$ the so-called shifted separatrix mapping has been proposed in which both time and energy variables are defined at the sections near the saddle points. A separatrix mapping to a section located in the middle of two successive saddle points for the periodi- 
cally driven pendulum has been also obtained in Refs. $[19,38]$. These mappings have been obtained from the conventional form of the separatrix mapping by a calculation of the increment of the energy to the corresponding sections. However, the mapping derived in Refs. $[19,38]$ is implicit in both time and energy variables, which makes it difficult for practical calculations.

In Refs. [48,49], the implicit symplectic mapping for canonical variables has been constructed for the perturbed Kepler problem. However the method which was employed for calculations of increments in time and energy over one phase rotation in phase space is not general and uses the specific features of the Kepler problem. Moreover, it requires us to impose additional assumptions on the dependence of these increments on time and energy variables in order to obtain area-preserving mappings.

In the present work, we develop a systematic and rigorous method to derive canonical symplectic mappings near the separatrix using the Hamilton-Jacobi method to construct mappings developed recently in Refs. [33,50]. The method is based on canonical transformations of variables in the Hamiltonian equations of motion. It allows one to derive directly a mapping near separatrix in canonical variables. A traditional separatrix mapping is obtained as a particular case of more general maps.

The content of the paper is as follows. In Sec. II, we formulate the problem, recall a conventional method to construct separatrix mappings due to Chirikov [3], and discuss the main shortcomings of these mappings. The method of canonical transformations of variables to derive mappings near separatrices is presented in Sec. III. Particularly, we construct mappings along a single saddle-saddle connection in terms of time and energy variables defined at sections of the phase space near the saddle points. Corresponding mappings at arbitrary sections of the phase space are constructed in Sec. IV. Methods to construct separatrix mappings are illustrated in Secs. V-IX for specific examples of Hamiltonian systems. Particularly, separatrix mappings describing a motion near the separatrices of a perturbed double-well potential and a periodically driven pendulum are constructed in Secs. V and VI, respectively. Separatrix mappings for Hamiltonian systems with a saddle point located at infinity are derived in Secs. VII and VIII. In Sec. VII, we constructed a mapping for the periodically driven Morse oscillator describing the process of stochastic excitation and dissociation of molecules in a microwave field. The Kepler map describing the process of chaotic ionization of highly excited hydrogen atoms in a microwave field is derived in Sec. VIII. Summary and conclusions are given in Sec. IX.

\section{CONVENTIONAL SEPARATRIX MAPPINGS}

For the sake of simplicity, we consider generic one degree of freedom Hamiltonian systems subjected to time-periodic perturbation. It is governed by the Hamilton equations

$$
\frac{d x}{d t}=\frac{\partial H}{\partial p}, \quad \frac{d p}{d t}=-\frac{\partial H}{\partial x},
$$

where $(x, p)$ are the canonical coordinate and momentum. The Hamiltonian $H(x, p, t)$ can be presented in the form
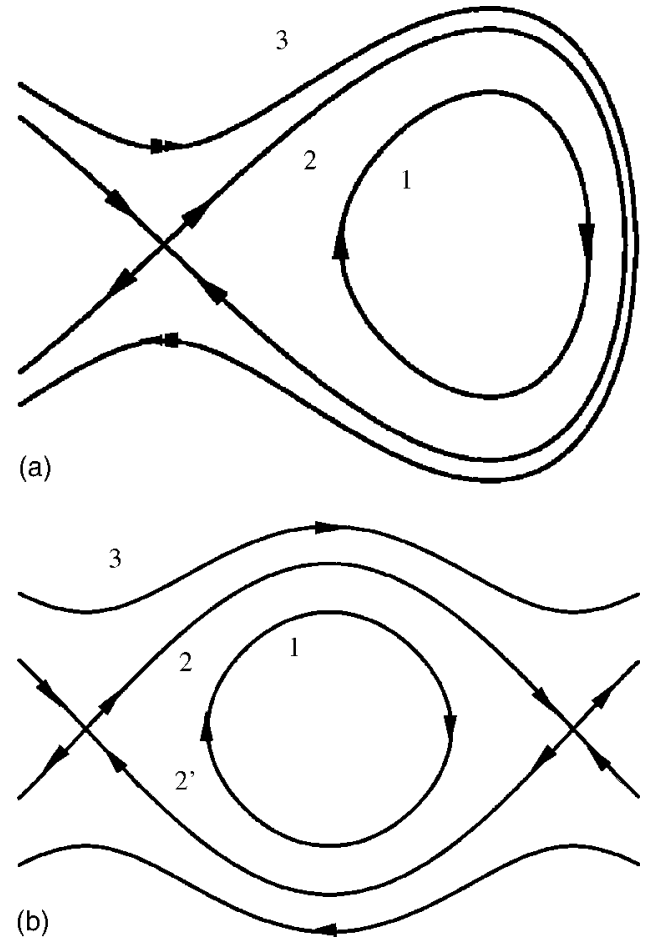

FIG. 1. Phase-space structure of system with separatrices: (a) homoclinic orbits (curve 2) and (b) heteroclinic orbits (curves 2 and $\left.2^{\prime}\right)$ connecting different saddles points.

$$
H(x, p, t)=H_{0}(x, p)+\epsilon H_{1}(x, p, t)
$$

where $H_{0}(x, p)$ is the unperturbed Hamiltonian, $H_{1}(x, p, t)$ is the time-dependent perturbation.

Suppose that an unperturbed system given by Hamiltonian $H_{0}(x, p)$ has one or more saddle points $\left(x_{s}, p_{s}\right)(s$ $=1,2, \ldots)$ at the same energetic level $E$, i.e., $E=H_{0}\left(x_{s}, p_{s}\right)$. These saddle points are connected by phase curves known as separatrices. These curves separate regions of phase space with different types of motion. Two examples of such saddlesaddle connections are shown in Fig. 1: (a) a saddle point is connected by itself by a so-called homoclinic orbit; (b) different saddle points are connected by a so-called heteroclinic orbit.

In typical Hamiltonian systems, separatrices are unstable to any small perturbations. In particular, a time-periodic perturbation destroys the separatrix, and the motion near the unperturbed separatrix becomes chaotic [59]. The domain of chaotic motion, the stochastic layer, is formed in the small vicinity of unperturbed separatrices.

Description of chaotic motion near separatrices by mappings was first considered in Refs. [3,53]. Particularly, in Ref. [53] a symplectic map for the periodically driven pendulum was introduced in terms of action-angle variables $(\vartheta, I)$, while a mapping in terms of time-energy variables $(t, H)$ was introduced in Ref. [3]. The last mapping is known as a separatrix mapping. We recall this map for the periodically driven pendulum described by the Hamiltonian 


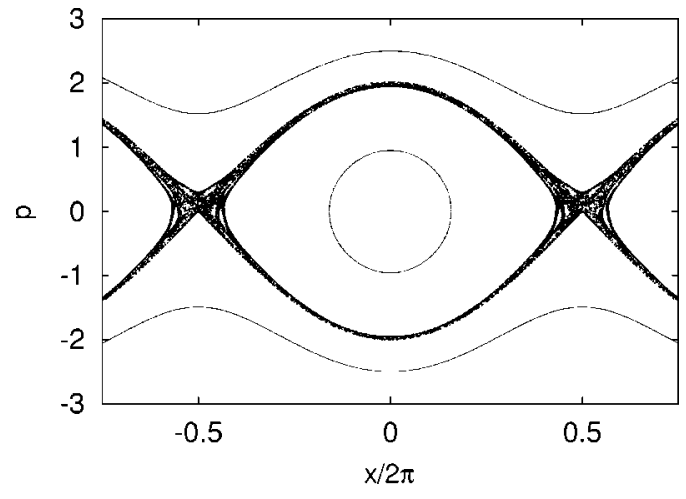

FIG. 2. Poincaré section of the system (3). One sees a stochastic layer near the unperturbed separatrix.

$$
H(x, p, t)=\frac{p^{2}}{2}-\omega_{0}^{2} \cos x+2 \epsilon \omega_{0}^{2} \cos x \cos \Omega t,
$$

where $\omega_{0}$ is the frequency of small oscillations, and $\epsilon$ and $\Omega$ represent the amplitude and the frequency of perturbation, respectively. The unperturbed system $(\epsilon=0)$ has elliptic fixed points at $(x=2 \pi n, p=0)$ and hyperbolic fixed points at $\left(x_{s}\right.$ $\left.=2 \pi(s+1 / 2), p_{s}=0\right)(n, s=0, \pm 1, \pm 2, \ldots)$. The separatrices (curve 2) connecting the saddle points $x_{s}, p_{s}$ with $q_{s \pm 1}, p_{s \pm 1}$ separate the trapped orbits $\left(-\omega_{0}^{2}<H<\omega_{0}^{2}\right)$ (curve 1) from the untrapped ones $\left(H>H_{s}=\omega_{0}^{2}\right)$ (curve 3). The period of trapped orbits $T(H)=2 \pi / \omega(H)$ has the following asymptotics near the separatrix $H=H_{s}$ :

$$
T(H)=\frac{1}{\omega_{0}} \ln \frac{32 \omega_{0}^{2}}{\left|H-\omega_{0}^{2}\right|}+O\left(\left|H-\omega_{0}^{2}\right|\right) \text { for } H \rightarrow \omega_{0}^{2}-0 .
$$

The orbits on the separatrices $\left(H=\omega_{0}^{2}\right)$ are

$$
\begin{gathered}
x_{s}^{( \pm)}(t)=4 \arctan \frac{\exp \left[ \pm \omega_{0}\left(t-t_{0}\right)\right]-1}{\exp \left[ \pm \omega_{0}\left(t-t_{0}\right)\right]+1}, \\
p_{s}^{( \pm)}(t)= \pm \frac{2 \omega_{0}}{\cosh \left[\omega_{0}\left(t-t_{0}\right)\right]},
\end{gathered}
$$

where the signs $( \pm)$ correspond to the upper (curve 2) and lower branches (curve $2^{\prime}$ ) of the separatrix, respectively, and $t_{0}$ is a time instant when the orbit crosses a midpoint between two sequential saddle points.

The perturbation $(\epsilon \neq 0)$ destroys the separatrix for any small amplitude of perturbation $\epsilon$ forming the stochastic layer near the unperturbed separatrix, as is shown in Fig. 2.

In Ref. [3], the separatrix mapping $\left.\left(t_{k}, H_{k}\right) \rightarrow\left(t_{k+1}, H_{k+1}\right)\right)$ is constructed calculating the increment of the time, $\Delta t\left(t_{k}, H_{k}\right)$, and energy, $\Delta H\left(t_{k}, H_{k}\right)$, over half of the phase rotation in phase space,

$$
\begin{gathered}
H_{k+1}=H_{k}+\Delta H\left(t_{k}, H_{k}\right), \\
t_{k+1}=t_{k}+\Delta t\left(t_{k}, H_{k}\right) .
\end{gathered}
$$

It is required that the mapping (6) should be area-preserving: $\left|\partial\left(H_{k+1}, t_{k+1}\right) / \partial\left(H_{k}, t_{k}\right)\right|=1$.
The energy increment, $\Delta H$, is found using the equation for the evolution of energy $H$,

$$
\frac{d H}{d t}=\frac{\partial H}{\partial t}=-2 \epsilon \Omega \omega_{0}^{2} \cos x \sin \Omega t,
$$

which is reduced to the Melnikov integral (see Ref. [3]),

$$
\begin{gathered}
\Delta H=-2 \epsilon \Omega \omega_{0}^{2} \int_{-\infty}^{\infty} \cos x_{s}(t) \sin \Omega t d t=-\omega_{0}^{2} W \sin \Omega t_{0}, \\
W=\epsilon \frac{\Omega^{2}}{\omega_{0}^{2}} \frac{4 \pi}{\sinh \left(\pi \Omega / 2 \omega_{0}\right)},
\end{gathered}
$$

taken along the unperturbed separatrix orbit $x_{s}(t)$ (5). The increment of time $\Delta t$ is equal to half of the period of oscillation $T(H)$ (4).

Identifying $t_{k}$ in Eq. (6) with $t_{0}$ and introducing the normalized variables $h=\left(H-\omega_{0}^{2}\right) / \omega_{0}^{2}$ and $\varphi=\Omega t_{0}$, the areapreserving map (6) is then presented in the form

$$
\begin{gathered}
h_{k+1}=h_{k}-W \sin \varphi_{k}, \\
\varphi_{k+1}=\varphi_{k}+\frac{\Omega}{\omega_{0}} \ln \frac{32}{\left|h_{k+1}\right|}, \bmod 2 \pi .
\end{gathered}
$$

which is known as the separatrix (or whisker) map.

The geometrical interpretation of the separatrix mapping was given by [17] as a return map of time and energy variables defined at the different sections in the phase space $(x, p)$ of the system. As we will see in Sec. III, the energy variable, $H$, is taken at the section near the saddle point, while the time variable, $t$, is in the section located in the middle between two consecutive saddle points (the sections $\Sigma_{s}$ and $\Sigma_{c}$ in Fig. 9, respectively). Therefore, the separatrix mapping (8) does not coincide with the definition of the Poincaré return map where all variables are defined at the same cross section of phase space. This means that the variables in the separatrix mapping are not canonical. This fact should be kept in mind when one compares the properties of the original continuous Hamiltonian system with those of the corresponding separatrix mapping.

As an example, one can recall the rescaling invariant property of the separatrix mapping (8) [41,42]. Since the second equation in Eqs. (8) is determined by module $2 \pi$, it is easy to see that the separatrix mapping is invariant with respect to the following transformation of perturbation parameter $\epsilon$ :

$$
\epsilon \rightarrow \epsilon^{\prime}=\lambda \epsilon,
$$

where the parameter $\lambda=\exp \left(2 \pi \omega_{0} / \Omega\right)$ depending only on the perturbation frequency $\Omega$ and the frequency $\omega_{0}$ of smallamplitude oscillations. This interesting property of the separatrix mapping, however, is not revealed in the phase space $(x, p)$ of the Hamiltonian system (3). As was shown in Refs. $[14,15]$, it does not exactly coincide with the rescaling invariance property of the Hamiltonian system near the hyperbolic saddle point. 


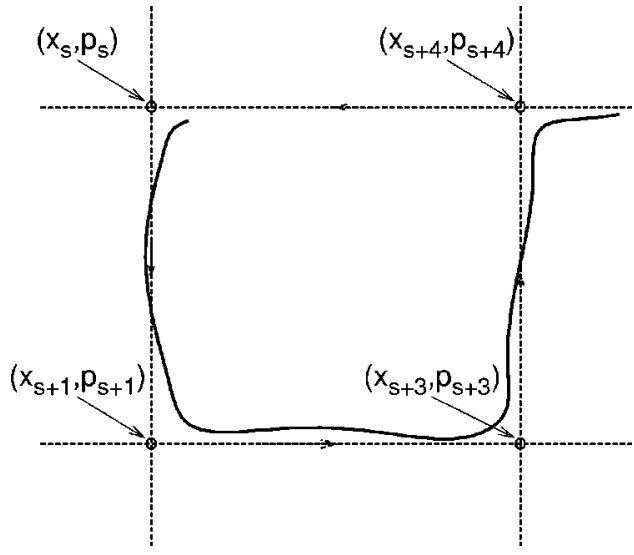

FIG. 3. Phase space of the system with several saddle points. Dotted curves describe the unperturbed orbit. A perturbed orbit is displayed by a solid curve.

\section{THE HAMILTON-JACOBI METHOD TO CONSTRUCT MAPS NEAR A SEPARATRIX}

Below, we present a rigorous derivation of symplectic mappings near the separatrix of general Hamiltonian systems using the Hamilton-Jacobi method developed in Ref. [50]. Furthermore, we refer to separatrix mappings in a broader sense as mappings near separatrices of arbitrary Hamiltonian systems. The idea of using this method to construct separatrix mappings was first mentioned in Ref. [33].

Suppose that the unperturbed system (1), (2) $(\epsilon=0)$ at a certain energy level $H_{0}(x, p)=$ const has a finite (or countable) number of saddle points and a corresponding number of saddle-saddle connections in phase space, as illustrated in Fig. 3. Let $\left(x_{s}, p_{s}\right)$ and $\left(x_{s+1}, p_{s+1}\right)$ be two consecutive saddle points with a heteroclinic connection, as shown in Fig. 4(a).

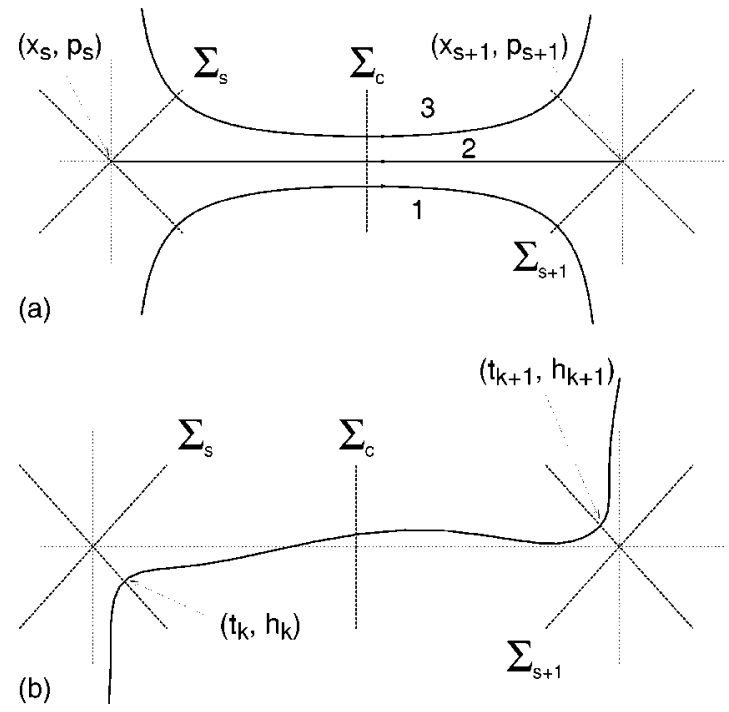

FIG. 4. (a) Phase curve in the neighborhood of the separatrix: curves 1 and 3 describe orbits up and down the separatrix, curve 2 is the separatrix. (b) Schematic view of the separatrix mapping. The solid curve describes the perturbed orbit, and the dotted curve is the unperturbed separatrix.
If the system has only one saddle point, then the points $\left(x_{s}, p_{s}\right)$ and $\left(x_{s+1}, p_{s+1}\right)$ coincide and a saddle-saddle connection is a homoclinic orbit. The phase-space curves 1 and 3 located on both sides of the separatrix describe different types of motion. We put $H=H_{S}=0$ on the separatrix.

In the phase plane $(x, p)$ we introduce cross sections $\Sigma_{c}$ and $\Sigma_{s}$, as shown in Fig. 4(a). The section $\Sigma_{c}$ consists of a segment perpendicular to the separatrix at the midpoint between saddle points. The section $\Sigma_{s}$ is located near saddle points $\left(x_{s}, p_{s}\right)$ and consists of two perpendicular segments with the crossing point at $\left(x_{s}, p_{s}\right)$. Both segments of $\Sigma_{s}$ are also perpendicular to unperturbed phase curves.

We define action-angle variables, $(I, \vartheta)$, for the unperturbed motion in the following way. They are introduced as integrals,

$$
I=\frac{1}{2 \pi} \int_{C} p(x ; H) d x, \quad \vartheta=\frac{\partial}{\partial I} \int^{x} p\left(x^{\prime} ; H\right) d x^{\prime},
$$

where $C$ is the segment of the phase-space curve of constant $H=H_{0}(x, p)$ located between two consecutive crossing points with the sections $\Sigma_{s}$ and $\Sigma_{s+1}$. Introduced in such a way, the action variable $I(H)$ is a continuous function of energy $H$ while it crosses the separatrix. We will set $\vartheta=0(\bmod 2 \pi)$ at the section $\Sigma_{c}$, and $\vartheta=\mp \pi(\bmod 2 \pi)$ at the sections $\Sigma_{s}$ and $\Sigma_{s+1}$, respectively.

In typical Hamiltonian systems, any small time-periodic perturbation destroys the separatrix, and orbits wobble around the unperturbed separatrix [see Fig. 4(b)]. Let $t_{k}$ and $h_{k}$ be a time instant and an energy of the system when the orbit crosses the section $\Sigma_{s}$. Index $k$ stands for the iteration number. We intend to construct the map

$$
\left(t_{k+1}, h_{k+1}\right)=\hat{M}_{s+1, s}\left(t_{k}, h_{k}\right),
$$

connecting the crossing point $\left(t_{k}, h_{k}\right)$ at the section $\Sigma_{s}$ with the corresponding point $\left(t_{k+1}, h_{k+1}\right)$ at $\Sigma_{s+1}$, where $h_{k}$ stands for the value of energy $H$ at the section $\Sigma_{s}$. The geometrical scheme of the mapping is shown in Fig. 4(b). The change of the angular variable $\vartheta$ over one step of the map (11) is equal to $\Delta \vartheta \equiv \vartheta_{k+1}-\vartheta_{k}=2 \pi$.

Suppose that the system has $N_{\text {sep }}$ independent saddlesaddle connections. Then there exist $N_{\text {sep }}$ independent mappings (11) which completely determine the dynamics of a Hamiltonian system. The sequence of mappings $\hat{M}_{s+1, s}$ depends on the topology of saddle-saddle connections in phase space and the trajectory of motion. Below, we develop a general method to construct the full set of mappings. Specific examples will be illustrated in the next sections.

\section{A. Mapping along a single separatrix}

Below, we construct the mapping (11) along a single saddle-saddle connection. For this purpose one could use the formulation of Hamiltonian equations with the angle variable, $\vartheta$, as an independent timelike variable. However, this approach fails near the separatrix where the frequency of motion $\omega(H)=d H_{0} / d I \rightarrow 0$ (or $d I / d H_{0} \rightarrow \infty$ ). This singularity does not allow us to invert Hamiltonian $H(I, \vartheta, t)$ with re- 
spect to the action variable $I$ near the separatrix. To avoid this difficulty, we will use another approach.

We use the formulation of Hamiltonian equations in the extended phase space $\left(t, x, p_{0}, p\right)$, which includes also time $t$ and "energy" $p_{0}=-H$ conjugated to time. The equations of motion in the space of action-angle and time-energy variables $\left(t, \vartheta, p_{0}, I\right)$ are

$$
\begin{aligned}
& \frac{d t}{d \tau}=\frac{\partial \mathcal{H}}{\partial p_{0}}, \quad \frac{d p_{0}}{d \tau}=-\frac{\partial \mathcal{H}}{\partial t}, \\
& \frac{d \vartheta}{d \tau}=\frac{\partial \mathcal{H}}{\partial I}, \quad \frac{d I}{d \tau}=-\frac{\partial \mathcal{H}}{\partial \vartheta},
\end{aligned}
$$

with the Hamiltonian function

$$
\mathcal{H}\left(t, \vartheta, p_{0}, I\right)=H_{0}(I)+p_{0}+\varepsilon \mathcal{H}_{1}\left(t, \vartheta, p_{0}\right)=0,
$$

where $\mathcal{H}_{1}\left(t, \vartheta, p_{0}\right) \equiv H_{1}\left(I\left(-p_{0}\right), \vartheta, t\right)$ is the perturbed part of the Hamiltonian. Here $\tau$ is an independent timelike variable. Note that in Eq. (13), the perturbation $\mathcal{H}_{1}$ is chosen as the function of energy $H=-p_{0}$.

Suppose that the orbit crosses the section $\Sigma_{s}$ at $\tau=\tau_{k}$ and the next section $\Sigma_{s+1}$ at $\tau=\tau_{k+1}$. First, we construct the mapping in the extended phase space, i.e.,

$$
\left(t_{k+1}, \vartheta_{k+1}, h_{k+1}, I_{k+1}\right)=\hat{M}\left(t_{k}, \vartheta_{k}, h_{k}, I_{k}\right),
$$

where $\left(t_{k}, \vartheta_{k}, h_{k}, I_{k}\right) \equiv\left(t\left(\tau_{k}\right), \vartheta\left(\tau_{k}\right),-p_{0}\left(\tau_{k}\right), I\left(\tau_{k}\right)\right)$. From the geometry of mapping illustrated in Fig. 4(b) it follows that we should impose constraints on the angle variable $\vartheta$ : $\vartheta_{k}=-\pi$ and $\vartheta_{k+1}=\pi$.

\section{B. Mappings}

According to the results of Ref. [50], the map (14) for the arbitrary time step $\Delta \tau=\tau_{k+1}-\tau_{k}$ can be presented in the following symplectic form:

$$
\begin{gathered}
J_{k}=I_{k}-\epsilon \frac{\partial S^{(k)}}{\partial \vartheta_{k}}, \\
\Theta_{k}=\vartheta_{k}+\epsilon \frac{\partial S^{(k)}}{\partial J_{k}}, \\
\bar{\Theta}_{k}=\Theta_{k}+w\left(\mathcal{H}_{k}, J_{k}, \epsilon\right)\left(\tau_{k+1}-\tau_{k}\right), \\
I_{k+1}=J_{k}+\epsilon \frac{\partial S^{(k+1)}}{\partial \vartheta_{k+1}}, \\
\vartheta_{k+1}=\bar{\Theta}_{k+1}-\epsilon \frac{\partial S^{(k+1)}}{\partial J_{k}},
\end{gathered}
$$

for action-angle variables $(\vartheta, I)$, and

$$
\begin{gathered}
\mathcal{H}_{k}=h_{k}+\epsilon \frac{\partial S^{(k)}}{\partial t_{k}}, \\
\mathcal{T}_{k}=t_{k}-\epsilon \frac{\partial S^{(k)}}{\partial \mathcal{H}_{k}},
\end{gathered}
$$

$$
\begin{gathered}
\overline{\mathcal{T}}_{k}=T_{k}+w_{t}\left(\mathcal{H}_{k}, J_{k}, \epsilon\right)\left(\tau_{k+1}-\tau_{k}\right), \\
h_{k+1}=\mathcal{H}_{k}-\epsilon \frac{\partial S^{(k+1)}}{\partial t_{k+1}}, \\
\vartheta_{k+1}=\overline{\mathcal{T}}_{k}+\epsilon \frac{\partial S^{(k+1)}}{\partial \mathcal{H}_{k}},
\end{gathered}
$$

for the time-energy variables $\left(t, h=-p_{0}\right)$. Here

$$
\begin{gathered}
S^{(k)} \equiv S\left(t_{k}, \vartheta_{k}, J_{k}, H_{k}, \tau_{k}, \tau_{0}, \epsilon\right), \\
S^{(k+1)} \equiv S\left(t_{k+1}, \vartheta_{k+1}, J_{k}, H_{k}, \tau_{k+1}, \tau_{0}, \epsilon\right),
\end{gathered}
$$

are values of the generating function $S\left(t, \vartheta, J, \mathcal{H}, \tau, \tau_{0}, \epsilon\right)$ at $\tau=\tau_{k}$ and $\tau=\tau_{k+1}$, respectively. For the Hamiltonian system (12), (13) it obeys the Hamilton-Jacobi equation

$$
\mathcal{H}\left(t, \vartheta, P_{0}+\epsilon \frac{\partial S}{\partial t}, J+\epsilon \frac{\partial S}{\partial \vartheta}\right)+\epsilon \frac{\partial S}{\partial \tau}=\bar{H}\left(P_{0}, J, \epsilon\right)
$$

in the time interval $\tau_{k}<\tau<\tau_{k+1}$ satisfying the initial condition $\left.S\right|_{\tau=\tau_{0}}=0$ at the time instant $\tau=\tau_{0}$. The time $\tau_{0}$ is a free parameter lying in the interval $\tau_{k}<\tau_{0}<\tau_{k+1}$. The new Hamiltonian $\bar{H}\left(P_{0}, J, \epsilon\right)$ depends only on new "action" variables $\left(P_{0}, J\right)$. In Eqs. (15) and (16), $w(\mathcal{H}, J, \epsilon)$ $=\partial \bar{H}\left(P_{0}, J, \epsilon\right) / \partial J$ and $w_{t}(\mathcal{H}, J, \epsilon)=\partial \bar{H}\left(P_{0}, J, \epsilon\right) / \partial P_{0}$ are the frequencies of perturbed motion. (Recall that $\mathcal{H}=-P_{0}$.)

\section{Perturbation series for the generating function}

The symplectic forms (15), (16) of the mapping (14) are general, and they are independent of assumptions of smallness of perturbation parameter $\epsilon$. Solutions of Eq. (17) in a finite time interval $\tau_{k}<\tau<\tau_{k+1}$ satisfying the condition $\left.S\right|_{\tau=\tau_{0}}=0$ can be found using a perturbation method by expanding Eq. (17), the new Hamiltonian $\bar{H}\left(P_{0}, J, \epsilon\right)$, and the generating function $S$ in series of powers of small perturbation parameter $\epsilon$,

$$
\bar{H}\left(P_{0}, J, \epsilon\right)=\bar{H}_{0}\left(J, P_{0}\right)+\epsilon \bar{H}_{1}\left(J, P_{0}\right)+\epsilon^{2} \bar{H}_{2}\left(J, P_{0}\right)+\cdots,
$$

where $\bar{H}_{0}\left(J, P_{0}\right)=H_{0}(J)+P_{0}$, and

$$
\begin{aligned}
S\left(t, \vartheta, J, \mathcal{H}, \tau, \tau_{0} ; \epsilon\right)= & S_{1}\left(t, \vartheta, J, \mathcal{H}, \tau, \tau_{0}\right) \\
& +\epsilon S_{2}\left(t, \vartheta, J, \mathcal{H}, \tau, \tau_{0}\right)+\cdots .
\end{aligned}
$$

The equations for the expansion coefficients $S_{i}$ $\equiv S_{i}\left(t, \vartheta, J, \mathcal{H}, \tau, \tau_{0}\right)$ are

$$
\frac{\partial S_{1}}{\partial \tau}+\frac{\partial \bar{H}_{0}}{\partial J} \frac{\partial S_{1}}{\partial \vartheta}+\frac{\partial \bar{H}_{0}}{\partial P_{0}} \frac{\partial S_{1}}{\partial t}=\bar{H}_{1}\left(J, P_{0}\right)-\mathcal{H}_{1}(t, \vartheta, \mathcal{H}),
$$




$$
\begin{aligned}
\frac{\partial S_{j}}{\partial \tau}+\frac{\partial \bar{H}_{0}}{\partial J} \frac{\partial S_{j}}{\partial \vartheta}+\frac{\partial \bar{H}_{0}}{\partial P_{0}} \frac{\partial S_{j}}{\partial t} & =\bar{H}_{j}\left(J, P_{0}\right)-F_{j}\left(t, \vartheta, J, \mathcal{H}, \tau, \tau_{0}\right), \\
j & \geqslant 2,
\end{aligned}
$$

where $F_{j}\left(t, \vartheta, J, \mathcal{H}, \tau, \tau_{0}\right)$ is the polynomial functions of derivatives $\partial S_{1} / \partial t, \partial S_{1} / \partial \vartheta, \ldots, \partial S_{j-1} / \partial t, \partial S_{j-1} / \partial \vartheta$.

The leading term $\bar{H}_{0}\left(J, P_{0}\right)$ in the new Hamiltonian (18) is equal to the old unperturbed Hamiltonian $H_{0}\left(I, P_{0}\right)$ (13), i.e., $\bar{H}_{0}\left(J, P_{0}\right)=H_{0}(I)+P_{0}$. The perturbative corrections $\bar{H}_{j}\left(J, P_{0}\right)$ in Eq. (18) can be chosen equal to values of functions $\mathcal{H}_{1}(t, \vartheta, \mathcal{H}), \mathcal{F}_{j}\left(t, \vartheta, J, \mathcal{H}, \tau, \tau_{0}\right)$ as averaged over "angle" variables $t, \vartheta$, i.e.,

$$
\begin{aligned}
\bar{H}_{1}\left(J, P_{0}\right) & =\left\langle\mathcal{H}_{1}(t, \vartheta, \mathcal{H})\right\rangle_{t, \vartheta} \\
& =\frac{\Omega}{(2 \pi)^{2}} \int_{0}^{2 \pi} d \vartheta \int_{0}^{2 \pi / \Omega} d t \mathcal{H}_{1}(t, \vartheta, \mathcal{H}) \bar{H}_{j}\left(J, P_{0}\right) \\
& =\left\langle F_{j}\left(t, \vartheta, J, \mathcal{H}, \tau, \tau_{0}\right)\right\rangle_{t, \vartheta} \\
& =\frac{\Omega}{(2 \pi)^{2}} \int_{0}^{2 \pi} d \vartheta \int_{0}^{2 \pi / \Omega} d t F_{j}\left(t, \vartheta, J, \mathcal{H}, \tau, \tau_{0}\right),
\end{aligned}
$$

where $\Omega$ is a perturbation frequency.

Further, for the sake of simplicity we will suppose that the averaged quantity $\bar{H}_{1}\left(J, P_{0}\right)$ is equal to zero. Then in the first order of perturbation $\epsilon$, the generating function $S$ is determined by the integral (see Ref. [50])

$$
S_{1}\left(t, \vartheta, \mathcal{H}, J, \tau, \tau_{0}\right)=-\int_{\tau_{0}}^{\tau} \mathcal{H}_{1}\left(t\left(\tau^{\prime}\right), \vartheta\left(\tau^{\prime}\right),-\mathcal{H}\right) d \tau^{\prime},
$$

taken along the unperturbed orbit $\vartheta\left(\tau^{\prime}\right)=\omega(J)\left(\tau^{\prime}-\tau\right)+\vartheta(\tau)$, $t\left(\tau^{\prime}\right)=\tau^{\prime}$. In this case the corresponding frequencies $\omega(J, \epsilon)$ and $\omega_{t}(J, \epsilon)$ can be replaced by the unperturbed frequencies $\omega(J)=\partial \mathcal{H}_{0}\left(J, p_{0}\right) / \partial J$ and $\omega_{t}(J)=\partial \mathcal{H}_{0}\left(J, P_{0}\right) / \partial P_{0}=1$, where $\mathcal{H}_{0}\left(J, P_{0}\right)=H_{0}(J)+P_{0}$.

Taking into account that the first-order generating function $S_{1}(21)$ does not depend on the action variable $J$, and choosing the times $\tau_{k}$ and $\tau_{k+1}$ at the sections $\Sigma_{k}$ and $\Sigma_{k+1}$, the mapping (15) can be reduced to the simplified form

$$
\begin{gathered}
J_{k}=I_{k}-\epsilon \frac{\partial S^{(k)}}{\partial \vartheta_{k}}, \\
\vartheta_{k+1}=\vartheta_{k}+\omega\left(J_{k}\right)\left(\tau_{k+1}-\tau_{k}\right), \\
I_{k+1}=J_{k}+\epsilon \frac{\partial S^{(k+1)}}{\partial \vartheta_{k+1}} .
\end{gathered}
$$

Recalling that $\vartheta_{k+1}-\vartheta_{k}=2 \pi$, and using the second equation in Eq. (22), we obtain $\tau_{k+1}-\tau_{k}=2 \pi / \omega\left(J_{k}\right)$. Then the mapping (11) for time-energy $(t, H)$ variables is reduced to

$$
\mathcal{H}_{k}=h_{k}+\epsilon \frac{\partial S^{(k)}\left(t_{k}, \mathcal{H}_{k}\right)}{\partial t_{k}}
$$

$$
\begin{gathered}
\mathcal{T}_{k}=t_{k}-\epsilon \frac{\partial S^{(k)}\left(t_{k}, \mathcal{H}_{k}\right)}{\partial \mathcal{H}_{k}}, \\
\overline{\mathcal{T}}_{k}=\mathcal{T}_{k}+\tau_{k+1}-\tau_{k}=\mathcal{T}_{k}+\frac{2 \pi}{\omega\left(\mathcal{H}_{k}\right)}, \\
h_{k+1}=\mathcal{H}_{k}-\epsilon \frac{\partial S^{(k+1)}\left(t_{k+1}, \mathcal{H}_{k}\right)}{\partial t_{k+1}}, \\
t_{k+1}=\overline{\mathcal{T}}_{k}+\epsilon \frac{\partial S^{(k+1)}\left(t_{k+1}, \mathcal{H}_{k}\right)}{\partial \mathcal{H}_{k}},
\end{gathered}
$$

where

$$
\begin{gathered}
S^{(k)}\left(t_{k}, \mathcal{H}\right)=S_{1}\left(t_{k}, \vartheta=-\pi, \mathcal{H}, \tau=t_{k}, \tau_{0}\right), \\
S^{(k+1)}\left(t_{k+1}, \mathcal{H}\right)=S_{1}\left(t_{k+1}, \vartheta=\pi, \mathcal{H}, \tau=t_{k+1}, \tau_{0}\right),
\end{gathered}
$$

and $\omega\left(\mathcal{H}_{k}\right) \equiv \omega\left(J_{k}\right)$.

Equations (23) present the general form of the mapping (11) of time $(t)$ and energy $(H)$ variables in the first order of perturbation parameter $\epsilon$. The corresponding generating function $S$ of this map is determined by Eq. (21). The separatrix mapping can be obtained from Eqs. (23) and (21) in some limiting cases. By appropriately choosing the time parameter $\tau_{0}$ in Eq. (21), one can obtain different forms of the mapping.

In the mapping (23) we have neglected the second- and higher-order terms $S_{j}(j \geqslant 2)$ in the expansion of the generating functions (19). These neglected terms are small in comparison to the first-order term $S_{1}$ and have an order $\epsilon^{j} \ll 1(j$ $\geqslant 2$ ).

\section{The first-order generating function}

Consider a multifrequency perturbation with frequencies $\Omega_{n}$ and present the perturbed Hamiltonian $\mathcal{H}_{1}(\mathcal{H}, \vartheta(t), t)$ in Eq. (21) taken along unperturbed trajectory as a Fourier series,

$$
\mathcal{H}_{1}(\tau, \vartheta(\tau),-\mathcal{H})=\sum_{n} H_{n}(\mathcal{H}, \vartheta(\tau)) \cos \left[\Omega_{n} t(\tau)+\chi_{n}\right]
$$

where $\chi_{n}$ are the phases of perturbation. Suppose the orbit crosses the section $\Sigma_{c}$ at the time instant $\tau=t_{c}$ when the phase $\vartheta=0$, and present the Fourier coefficients as

$$
V_{n}\left(\mathcal{H}, \tau-t_{c}\right) \equiv H_{n}(\mathcal{H}, \vartheta(\tau)) .
$$

Taking into account that the unperturbed orbit is given by $t=\tau, \quad \vartheta\left(\tau^{\prime}\right)=\vartheta+\omega(\mathcal{H})\left(\tau^{\prime}-\tau\right)$, we find that $t_{c}=t-\vartheta / \omega(\mathcal{H})$. Then the generating function $S(t) \equiv S_{1}\left(t, \vartheta, \mathcal{H}, \tau, \tau_{0}\right)$ in the time interval $t_{k}<t, t_{c}<t_{k+1}$ can be reduced to

$$
S(t)=-\int_{\tau_{0}}^{t} \sum_{n} V_{n}\left(\mathcal{H}, t^{\prime}-t+\frac{\vartheta}{\omega(\mathcal{H})}\right) \cos \left(\Omega_{n} t^{\prime}+\chi_{n}\right) d t^{\prime},
$$




$$
=\operatorname{Re} \sum_{n} R_{n}(\mathcal{H}, \vartheta, t) \exp \left[i \Omega_{n}\left(t-\frac{\vartheta}{\omega(\mathcal{H})}\right)+i \chi_{n}\right],
$$

where

$$
R_{n}(\mathcal{H}, \vartheta, t)=\int_{\vartheta / \omega(\mathcal{H})}^{\tau_{0}+\vartheta / \omega(\mathcal{H})-t} V_{n}(\mathcal{H}, \tau) e^{i \Omega_{n} \tau} d \tau
$$

At the limits $t \rightarrow t_{k}+0, \quad \vartheta \rightarrow-\pi \quad$ and $\quad t \rightarrow t_{k+1}-0=t_{k}$ $+2 \pi / \omega(\mathcal{H}), \vartheta \rightarrow \pi$ we have

$$
\begin{aligned}
S^{(k)}\left(t_{k}, \mathcal{H}\right)=\sum_{n}\left[K_{n}^{+}(\mathcal{H})\right. & \left.\cos \Phi_{n}^{+}\left(t_{k}, \mathcal{H}\right)-L_{n}^{+}(\mathcal{H}) \sin \Phi_{n}^{+}\left(t_{k}, \mathcal{H}\right)\right] \\
S^{(k+1)}\left(t_{k+1}, \mathcal{H}\right)= & \sum_{n}\left[K_{n}^{-}(\mathcal{H}) \cos \Phi_{n}^{-}\left(t_{k+1}, \mathcal{H}\right)\right. \\
& \left.-L_{n}^{-}(\mathcal{H}) \sin \Phi_{n}^{-}\left(t_{k+1}, \mathcal{H}\right)\right]
\end{aligned}
$$

where

$$
\Phi_{n}^{ \pm}(t, \mathcal{H})=\Omega_{n}\left(t \pm \frac{\pi}{\omega(\mathcal{H})}\right)+\chi_{n},
$$

and $K_{n}^{ \pm}(\mathcal{H}), L_{n}^{ \pm}(\mathcal{H})$ are real and imaginary parts of the integrals $R_{n}^{ \pm}(\mathcal{H})=K_{n}^{ \pm}(\mathcal{H})+i L_{n}^{ \pm}(\mathcal{H})$, respectively, defined as

$$
\begin{aligned}
R_{n}^{+}(\mathcal{H}) & \equiv R_{n}\left(\mathcal{H}, \vartheta=-\pi, t=t_{k}\right) \\
& =\int_{-\pi / \omega(\mathcal{H})}^{\tau_{0}-t_{k}-\pi / \omega(\mathcal{H})} V_{n}(\mathcal{H}, \tau) e^{i \Omega_{n} \tau} d \tau, \\
R_{n}^{-}(\mathcal{H}) & \equiv R_{n}\left(\mathcal{H}, \vartheta=\pi, t=t_{k+1}\right) \\
& =\int_{\pi / \omega(\mathcal{H})}^{\tau_{0}-t_{k+1}+\pi / \omega(\mathcal{H})} V_{n}(\mathcal{H}, \tau) e^{i \Omega_{n} \tau} d \tau .
\end{aligned}
$$

\section{E. Symmetric mappings}

We call the mapping (23) a symmetric map when the free parameter $\tau_{0}$ is taken exactly in the middle between $\tau_{k}$ and $\tau_{k+1}$, i.e., $\tau_{0}=\left(\tau_{k+1}+\tau_{k}\right) / 2=t_{k}+\pi / \omega(\mathcal{H})$. Then the integrals (30) take the form

$$
R_{n}^{ \pm}(\mathcal{H})=K_{n}^{ \pm}(\mathcal{H})+i L_{n}^{ \pm}(\mathcal{H})=\int_{\mp \pi / \omega(\mathcal{H})}^{0} V_{n}(\mathcal{H}, \tau) e^{i \Omega_{n} \tau} d \tau .
$$

The Fourier integrals (31) are taken along the unperturbed orbits of the system. In a particular case, when the orbits lie on the separatrix $(h=0)$ they coincide with the MelnikovArnold-type integrals of type (7) (see [3]). Indeed, at the limit $h \rightarrow 0$ the frequency $\omega(h) \rightarrow 0$ and the integrals (31) are reduced to

$$
\begin{aligned}
& R_{n}^{+}(0)=\int_{-\infty}^{0} V_{n}(0, \tau) e^{i \Omega_{n} \tau} d \tau, \\
& R_{n}^{-}(0)=-\int_{0}^{\infty} V_{n}(0, \tau) e^{i \Omega_{n} \tau} d \tau .
\end{aligned}
$$

It is easy to see that the symmetric map conserves an invariance of the Hamiltonian system with respect to time reversing, $t \rightarrow-t, H \rightarrow-H$, which is manifested in the invariance of mapping with respect to reversing the mapping sequence, $k \leftrightarrow k+1$.

\section{F. Nonsymmetric mappings}

Another form of mappings can be obtained by setting the free time parameter $\tau_{0}$ in Eq. (30) equal to $\tau_{k}$ or $\tau_{k+1}$. Consider first the case when $\tau_{0}=\tau_{k+1}$, the integrals $K_{n}^{(-)}$and $L_{n}^{(-)}$ in Eq. (30) vanish, and therefore $S^{(k+1)} \equiv 0, h_{k+1}=\mathcal{H}_{k}$. Then the mapping (23) is reduced to the mapping

$$
\begin{gathered}
h_{k+1}=h_{k}+\epsilon \frac{\partial S^{(k)}\left(t_{k}, h_{k+1}\right)}{\partial t_{k}}, \\
t_{k+1}=t_{k}+\frac{2 \pi}{\omega\left(h_{k+1}\right)}-\epsilon \frac{\partial S^{(k)}\left(t_{k}, h_{k+1}\right)}{\partial h_{k+1}},
\end{gathered}
$$

determined by only one generating function $S^{(k)}\left(t_{k}, h_{k+1}\right)$,

$$
\begin{aligned}
S^{(k)}\left(t_{k}, h_{k+1}\right)= & \sum_{n}\left[K_{n}\left(h_{k+1}\right) \cos \Phi_{n}^{+}\left(t_{k}, h_{k+1}\right)\right. \\
& \left.-L_{n}\left(h_{k+1}\right) \sin \Phi_{n}^{+}\left(t_{k}, h_{k+1}\right)\right],
\end{aligned}
$$

where $K(h)$ and $L(h)$ are the integrals,

$$
K_{n}(h)+i L_{n}(h)=\int_{-\pi / \omega(h)}^{\pi / \omega(h)} V_{n}(h, \tau) e^{i \Omega_{n} \tau} d \tau .
$$

On the other hand, putting $\tau_{0}=\tau_{k}$ we have $S^{(k)} \equiv 0$ and $h_{k}=\mathcal{H}_{k}$ since the integrals $K_{n}^{(+)} \equiv 0$ and $L_{n}^{(+)} \equiv 0$, and the mapping (23) is reduced to

$$
\begin{gathered}
h_{k+1}=h_{k}-\epsilon \frac{\partial S^{(k+1)}\left(t_{k+1}, h_{k}\right)}{\partial t_{k+1}}, \\
t_{k+1}=t_{k}+\frac{2 \pi}{\omega\left(h_{k}\right)}+\epsilon \frac{\partial S^{(k+1)}\left(t_{k+1}, h_{k}\right)}{\partial h_{k}},
\end{gathered}
$$

where $S^{(k+1)}\left(t_{k+1}, h_{k}\right)$ is given by

$$
\begin{aligned}
S^{(k+1)}\left(t_{k+1}, h_{k}\right)= & -\sum_{n}\left[K_{n}\left(h_{k}\right) \cos \Phi_{n}^{-}\left(t_{k+1}, h_{k}\right)\right. \\
& \left.-L_{n}\left(h_{k}\right) \sin \Phi_{n}^{-}\left(t_{k+1}, h_{k}\right)\right] .
\end{aligned}
$$

In Eqs. (34) and (37), the phases $\Phi_{n}^{ \pm}(t, h)$ are defined by Eq. (29). At the limit $h \rightarrow 0$, we have

$$
K_{n}(0)+i L_{n}(0)=\int_{-\infty}^{\infty} V_{n}(0, \tau) e^{i \Omega_{n} \tau} d \tau .
$$

We call the mappings (33), (36) nonsymmetric mappings since they are not invariant with respect to reversing the mapping sequence, $k \leftrightarrow k+1$.

\section{G. Simplified forms of mappings}

The symmetric mapping (23) with the generating functions (28) have a rather complicated form. It can be simpli- 
fied using the smallness of perturbation parameter $\epsilon$. Eliminating the intermediate variables $\mathcal{H}, \mathcal{T}$, we transform a set of Eqs. (23) into

$$
\begin{gathered}
h_{k+1}=h_{k}-\epsilon\left(\frac{\partial S^{(k+1)}}{\partial t_{k+1}}-\frac{\partial S^{(k)}}{\partial t_{k}}\right), \\
t_{k+1}=t_{k}+\frac{2 \pi}{\omega\left(\mathcal{H}_{k}\right)}+\epsilon\left(\frac{\partial S^{(k+1)}}{\partial \mathcal{H}_{k}}-\frac{\partial S^{(k)}}{\partial \mathcal{H}_{k}}\right) .
\end{gathered}
$$

Using Eqs. (23) and (28), one can show that

$$
\begin{aligned}
& \frac{2 \pi}{\omega\left(\mathcal{H}_{k}\right)}+\epsilon\left(\frac{\partial S^{(k+1)}}{\partial \mathcal{H}_{k}}-\frac{\partial S^{(k)}}{\partial \mathcal{H}_{k}}\right)=\frac{\pi}{\omega\left(h_{k}\right)}+\frac{\pi}{\omega\left(h_{k+1}\right)} \\
&+G\left(t_{k}, h_{k+1}, h_{k}\right)+O\left(\epsilon^{2}\right), \\
& \epsilon\left(\frac{\partial S^{(k+1)}}{\partial t_{k+1}}-\frac{\partial S^{(k)}}{\partial t_{k}}\right)=\epsilon F\left(t_{k}, h_{k+1}, h_{k}\right)+O\left(\epsilon^{2}\right),
\end{aligned}
$$

where

$$
\begin{aligned}
F\left(t_{k}, h_{k+1}, h_{k}\right)= & \sum_{n} \Omega_{n}\left[K_{n}\left(h_{k+1}\right) \sin \Phi_{n}^{+}\left(t_{k}, h_{k}\right)\right. \\
& \left.+L_{n}\left(h_{k+1}\right) \cos \Phi^{+}\left(t_{k}, h_{k}\right)\right], \\
G\left(t_{k}, h_{k+1}, h_{k}\right)= & \sum_{n}\left(\frac{d K_{n}\left(h_{k+1}\right)}{d h_{k+1}} \cos \Phi_{n}^{+}\left(t_{k}, h_{k}\right)\right. \\
& \left.-\frac{d L_{n}\left(h_{k+1}\right)}{d h_{k+1}} \sin \Phi^{+}\left(t_{k}, h_{k}\right)\right) .
\end{aligned}
$$

with the coefficients $K_{n}(h), L_{n}(h)$ determined by Eq. (35). Neglecting the terms of order $\epsilon^{2}$, one obtains

$$
\begin{gathered}
h_{k+1}=h_{k}-\epsilon F\left(t_{k}, h_{k+1}, h_{k}\right), \\
t_{k+1}=t_{k}+\frac{\pi}{\omega\left(h_{k}\right)}+\frac{\pi}{\omega\left(h_{k+1}\right)}+\epsilon G\left(t_{k}, h_{k+1}, h_{k}\right) .
\end{gathered}
$$

A straightforward calculation shows that $\left|\partial\left(h_{k+1}, t_{k+1}\right)\right|$ $\partial\left(h_{k}, t_{k}\right) \mid=1$, i.e., the mapping (41) is a symplectic. The mapping (41) is also invariant with respect to the time-reversing transformation, $k \leftrightarrow k+1$.

The mapping (41) can also be obtained from the nonsymmetric forms of the mappings (33), (36) using a similar procedure.

\section{H. A case of a thin stochastic layer}

Suppose that the stochastic layer formed near the unperturbed separatrix is sufficiently thin that the variation of energy $H$ about the separatrix energy $H_{s}=0$ is small. Then the integrals (31) in the generating functions (28) can be replaced by the Melnikov-Arnold-type integrals (32). Then the mapping (41) can be further simplified to

$$
\begin{aligned}
h_{k+1}= & h_{k}-\epsilon \sum_{n} \Omega_{n}\left\{K_{n} \sin \left[\Omega_{n}\left(t_{k}+\frac{\pi}{\omega\left(h_{k}\right)}\right)+\chi_{n}\right]\right. \\
& \left.+L_{n} \cos \left[\Omega_{n}\left(t_{k}+\frac{\pi}{\omega\left(h_{k}\right)}\right)+\chi_{n}\right]\right\},
\end{aligned}
$$

$$
t_{k+1}=t_{k}+\frac{\pi}{\omega\left(h_{k}\right)}+\frac{\pi}{\omega\left(h_{k+1}\right)},
$$

where $K_{n}$ and $L_{n}$ are the integrals defined by Eq. (38).

The mapping (42) was first introduced in $[14,15]$ to study magnetic-field lines in plasmas. It is called a shifted separatrix mapping since it was obtained from the conventional separatrix mapping by shifting the phases $\Omega t_{k}$ (see Sec. IV A.

This mapping was instrumental in explaining the rescaling invariance property of Hamiltonian systems near saddle points (see Ref. [46] and references therein). This property consists of the fact that the phase space of the Hamiltonian systems near the hyperbolic saddle points subjected to a small time-periodic perturbation is invariant with respect to the following transformations of the perturbation parameter $\epsilon$, its phase $\chi$, and the phase-space coordinates $x, p$ :

$$
\begin{gathered}
\epsilon \rightarrow \epsilon^{\prime}=\lambda \epsilon, \quad \chi \rightarrow \chi^{\prime}=\chi+\pi, \\
x \rightarrow x^{\prime}=\lambda^{1 / 2} x, \quad p \rightarrow p^{\prime}=\lambda^{1 / 2} p,
\end{gathered}
$$

where $\lambda$ is a universal parameter determined by the frequency of perturbation $\Omega$ and the expansion coefficient $\gamma$ of unperturbed Hamiltonian $H_{0}(x, p)$ near saddle points: $H_{0}(x, p) \approx \gamma x p$ (in an appropriate coordinate system), i.e., $\lambda$ $=\exp (2 \pi \gamma / \Omega)$. [For the pendulum it coincides with the parameter $\lambda$ in Eq. (9).]

The mapping (42) describes this property of Hamiltonian systems. Indeed, in Hamiltonian systems with hyperbolic saddle points the frequency of motion $\omega(h)$ has the following universal asymptotics near the separatrix: $\omega(h)=\gamma / \ln (A /|h|)$, where $A$ is a constant parameter. When the perturbation $H_{1}(x, p, t)$ in Eqs. (2) and (25) is periodic in time with the frequency $\Omega$, i.e., $\Omega_{n}=n \Omega$, the mapping (42) is invariant with respect to the following transformations:

$$
\begin{gathered}
\epsilon \rightarrow \epsilon^{\prime}=\lambda \epsilon, \quad \chi_{n} \rightarrow \chi_{n}^{\prime}=\chi_{n}+n \pi, \\
h \rightarrow h^{\prime}=\lambda h,
\end{gathered}
$$

which is equivalent to transformations (43).

\section{MAPPING AT ARBITRARY SECTIONS OF THE PHASE SPACE}

In some applications it is necessary to construct mappings with variables defined at arbitrary sections of the phase space. These mappings can also be constructed similar to the ones presented above. However, in general, a construction of such mappings is not as straightforward as the mappings to the sections $\Sigma_{s}$ along the single saddle-saddle connection (see Fig. 4). In the latter case, the mapping (11) is determined only by orbits between sections $\Sigma_{s}$ and $\Sigma_{s+1}$. In order to obtain a mapping (11) where the variables $(t, H)$ are defined, for instance, at the sections $\Sigma_{c}$ (see Fig. 4), one needs to know the topology of all saddle-saddle connections.

Figure 5 illustrates the example of the mapping to the sections $\Sigma_{c}$. Suppose the orbit crosses the section $\Sigma_{c}$ at $\left(t_{k}, h_{k}\right)$. Because of a sensitive dependence of orbits near the 


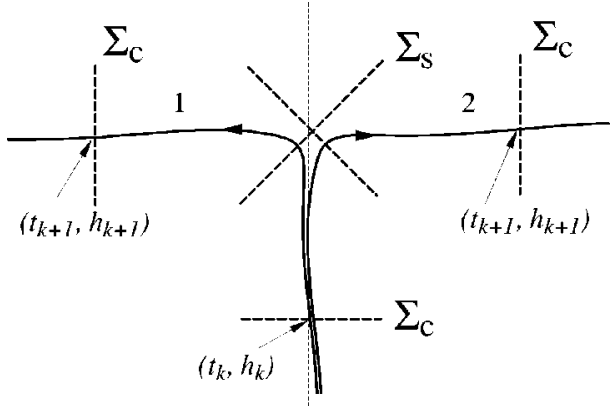

FIG. 5. Geometrical illustration of the mapping to the sections $\Sigma_{c}$. Solid curves 1 and 2 describe the perturbed orbits, and dotted curves are the unperturbed separatrices.

separatrix on their initial condition, the next crossing point $\left(t_{k+1}, h_{k+1}\right)$ may lie on the section $\Sigma_{c}$ either on the left side for the orbits of type 1 or on the right side for the orbits of type 2. The direction of orbits is determined by the value of energy variable $H$ at the crossing point of the orbit with $\Sigma_{s}$. Depending on the condition $H>H_{s}=0$ or $H<H_{s}=0$, the orbit may cross the section $\Sigma_{c}$ on the left-hand side or on the right-hand side. In this sense the mapping, in general, should be constructed in an algorithmic way. Below, we construct corresponding mapping for the system with a single saddle point and only one saddle-saddle connection. The case of a system with more than one saddle-saddle connection will be considered in Sec. VI B for the periodically driven pendulum.

\section{A. A symmetric mapping to the section $\mathbf{\Sigma}_{c}$}

The phase space of such a system is shown in Fig. 6. The hyperbolic saddle point is located at $\left(x_{s}=0, p_{s}=0\right)$. The dotted curve describes the unperturbed separatrix. The orbit reflects from the rigid border $x=0$ changing the sign of momentum $p$. Then the mapping $\left(t_{k}, h_{k}\right) \rightarrow\left(t_{k+1}, h_{k+1}\right)$ defines the Poincaré return map to the section $\Sigma_{c}$. It has a general form (23) with the generating function (26), (27). We should put $\vartheta=0(\bmod 2 \pi)$ at both time instants $t=t_{k}$ and $t=t_{k+1}$. For the generating functions $S^{(k)}$ and $S^{(k+1)}$ of the mapping (23), we have

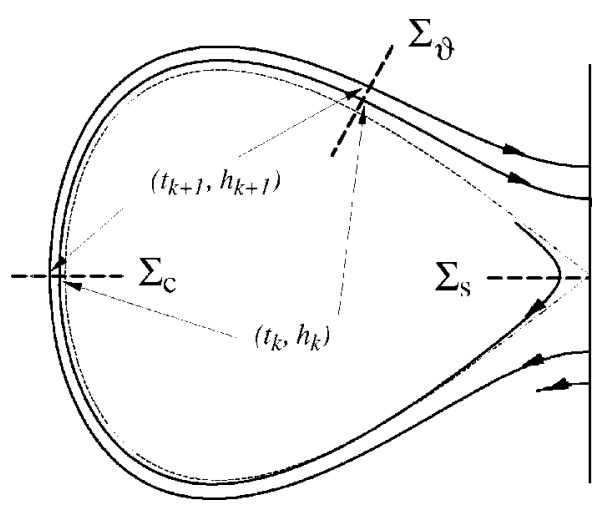

FIG. 6. Geometry of the mapping to the sections $\Sigma_{c}$ and $\Sigma_{\vartheta}$ in the system with the single saddle point.

$$
\begin{aligned}
S^{(k)}\left(t_{k}, \mathcal{H}_{k}\right)= & -\sum_{n}\left[K_{n}^{+}\left(\mathcal{H}_{k}\right) \cos \left(\Omega_{n} t_{k}+\chi_{n}\right)\right. \\
& \left.-L_{n}^{+}\left(\mathcal{H}_{k}\right) \sin \left(\Omega_{n} t_{k}+\chi_{n}\right)\right], \\
S^{(k+1)}\left(t_{k+1}, \mathcal{H}_{k}\right)= & \sum_{n}\left[K_{n}^{-}\left(\mathcal{H}_{k}\right) \cos \left(\Omega_{n} t_{k+1}+\chi_{n}\right)\right. \\
& \left.-L_{n}^{-}\left(\mathcal{H}_{k}\right) \sin \left(\Omega_{n} t_{k+1}+\chi_{n}\right)\right],
\end{aligned}
$$

where $K_{n}^{ \pm}(\mathcal{H}), L_{n}^{ \pm}(\mathcal{H})$ are the integrals of Eq. (31).

Equations (23) with the generating functions (45) determine the corresponding map. The first set of this mapping is implicit in energy variable $\mathcal{H}$, and the last set is implicit in time $t_{k+1}$.

The mapping can be significantly simplified if the stochastic layer is sufficiently thin. In this case, one can replace the integrals (31) by their values at $\mathcal{H}=0$,

$$
K_{n}^{ \pm}(0)+i L_{n}^{ \pm}(0)=\int_{0}^{ \pm \infty} V_{n}(0, \tau) e^{i \Omega_{n} \tau} d \tau .
$$

In this approximation the generating function (45) does not depend on energy variable $\mathcal{H}$, and the mapping (23) takes the simplified form

$$
\begin{gathered}
\mathcal{H}_{k}=h_{k}+\epsilon \frac{\partial S^{(k)}}{\partial t_{k}}, \\
t_{k+1}=t_{k}+\frac{2 \pi}{\omega\left(\mathcal{H}_{k}\right)}, \\
h_{k+1}=\mathcal{H}_{k}-\epsilon \frac{\partial S^{(k+1)}}{\partial t_{k+1}},
\end{gathered}
$$

or

$$
\mathcal{H}_{k}=h_{k}-\epsilon \sum_{n} \Omega_{n}\left[K_{n}^{+} \sin \left(\Omega_{n} t_{k}+\chi_{n}\right)+L_{n}^{+} \cos \left(\Omega_{n} t_{k}+\chi_{n}\right)\right],
$$

$$
\begin{gathered}
t_{k+1}=t_{k}+\frac{2 \pi}{\omega\left(\mathcal{H}_{k}\right)}, \\
h_{k+1}=\mathcal{H}_{k}+\epsilon \sum_{n} \Omega_{n}\left[K_{n}^{-} \sin \left(\Omega_{n} t_{k+1}+\chi_{n}\right)\right. \\
\left.+L_{n}^{-} \cos \left(\Omega_{n} t_{k+1}+\chi_{n}\right)\right] .
\end{gathered}
$$

This map determines the Poincaré return map of energy $(H)$ and time $(t)$ variables to the section $\Sigma_{c}$.

The map (48) can also be written in the form of mapping $\left(t_{k}, \mathcal{H}_{k-1}\right) \rightarrow\left(t_{k+1}, \mathcal{H}_{k}\right)$,

$$
\mathcal{H}_{k}=\mathcal{H}_{k-1}+\epsilon \sum_{n} \Omega_{n}\left[K_{n} \sin \left(\Omega_{n} t_{k}+\chi_{n}\right)+L_{n} \cos \left(\Omega_{n} t_{k}+\chi_{n}\right)\right],
$$




$$
t_{k+1}=t_{k}+\frac{2 \pi}{\omega\left(\mathcal{H}_{k}\right)},
$$

where the coefficients $K_{n}=K_{n}^{+}-K_{n}^{-}$and $L_{n}=L_{n}^{+}-L_{n}^{-}$are determined by the Melnikov-Arnold-type integrals (38).

Equations (49) coincide with the conventional form of the separatrix map in which the energy $(H)$ and time $(t)$ are defined at different sections of the phase space. Indeed, the intermediate variable $\mathcal{H}$ coincides with the energy taken at the section $\Sigma_{s}$, while $t$ is on the section $\Sigma_{c}$.

One should also note that the mapping (42) at the section $\Sigma_{s}$ can be formally derived from the mapping (49) by shifting the time variable $t_{k}$ from the section $\Sigma_{c}$ to the section $\Sigma_{s}$. Since the phase difference between these sections is $\pi$, the time difference along the unperturbed orbit is equal to $2 \pi / \omega(\mathcal{H})$. Taking into account that the energy variable $\mathcal{H}_{k}$ in the map (49) coincides with the one at the section $\Sigma_{s}$ and replacing $\mathcal{H}_{k-1} \rightarrow h_{k}, t_{k} \rightarrow t_{k}+\pi / \omega\left(h_{k}\right)$, we obtain the mapping (42).

\section{B. Mapping to the section $\Sigma_{\vartheta}$}

Now we consider the mapping to the arbitrary section $\Sigma_{\vartheta}$ on phase space. The schematic view of the section $\Sigma_{\vartheta}$ is shown in Fig. 6. It is specified by the phase $\vartheta=$ const $(-\pi<\vartheta<\pi)$, and consists of the segment of a straight line that can be reached from the section $\Sigma_{c}$ in time $\Delta t(h)$ $=|\vartheta| / \omega(h)$ along unperturbed phase curves about the separatrix. The return map $\left(t_{k}, h_{k}\right) \rightarrow\left(t_{k+1}, h_{k+1}\right)$, where the variables $\left(t_{k}, h_{k}\right)$ are at the section $\Sigma_{\vartheta}$, is given by Eqs. (23). According to Eqs. (26), (27), the generating functions $S^{(k)}, S^{(k+1)}$ are

$$
\begin{gathered}
S^{(k)}=\sum_{n}\left[K_{n}^{+}(\mathcal{H}, \vartheta) \cos \Phi_{n}\left(t_{k}, \mathcal{H}\right)-L_{n}^{+}(\mathcal{H}, \vartheta) \sin \Phi_{n}\left(t_{k}, \mathcal{H}\right)\right] \\
S^{(k+1)}=\sum_{n}\left[K_{n}^{-}(\mathcal{H}, \vartheta) \cos \Phi_{n}\left(t_{k+1}, \mathcal{H}\right)\right. \\
\left.-L_{n}^{-}(\mathcal{H}, \vartheta) \sin \Phi_{n}\left(t_{k+1}, \mathcal{H}\right)\right]
\end{gathered}
$$

where

$$
\Phi_{n}(t, H)=\Omega_{n}\left(t-\frac{\vartheta}{\omega(H)}\right)+\chi_{n},
$$

and $K_{n}^{ \pm}(\mathcal{H})+i L_{n}^{ \pm}(\mathcal{H})=R_{n}^{ \pm}(\mathcal{H})$

$$
\begin{gathered}
R_{n}^{+}(\mathcal{H}, \vartheta) \equiv R_{n}^{+}\left(\mathcal{H}, \vartheta, t=t_{k}\right)=\int_{\vartheta / \omega(\mathcal{H})}^{\pi / \omega(\mathcal{H})} V_{n}(\mathcal{H}, \tau) e^{i \Omega_{n} \tau} d \tau, \\
R_{n}^{-}(\mathcal{H}, \vartheta) \equiv R_{n}^{-}\left(\mathcal{H}, \vartheta, t=t_{k+1}\right)=\int_{\vartheta / \omega(\mathcal{H})}^{\pi / \omega(\mathcal{H})} V_{n}(\mathcal{H}, \tau) e^{i \Omega_{n} \tau} d \tau .
\end{gathered}
$$

Consider the case of the thin stochastic layer taking the limiting case $\mathcal{H} \rightarrow 0$. Then the integrals (52) have the following limits. For the value of the phase $\vartheta$ in the interval $-\pi<\vartheta<0$, we have $R_{n}^{+}(0)=K_{n}(0)+i L_{n}(0)$ determined by the integral (38) and $R_{n}^{-}=0$. Therefore, the generating function $S^{(k+1)}=0$ and the mapping (23) is reduced to

$$
\begin{gathered}
h_{k+1}=h_{k}+\epsilon \frac{\partial S\left(t_{k}, h_{k+1}\right)}{\partial t_{k}}, \\
t_{k+1}=t_{k}+\frac{2 \pi}{\omega\left(h_{k+1}\right)}-\epsilon \frac{\partial S\left(t_{k}, h_{k+1}\right)}{\partial h_{k+1}},
\end{gathered}
$$

with the generating function

$$
S(t, h)=\sum_{n}\left[K_{n}(0) \cos \Phi_{n}(t, h)-L_{n}(0) \sin \Phi_{n}(t, h)\right] .
$$

Similarly, for $0<\vartheta<\pi$ we obtain $R_{n}^{+}=0$ and $R_{n}^{-}(0)$ $=-K_{n}(0)-i L_{n}(0)$. The mapping becomes

$$
\begin{gathered}
h_{k+1}=h_{k}+\epsilon \frac{\partial S\left(t_{k+1}, h_{k}\right)}{\partial t_{k+1}}, \\
t_{k+1}=t_{k}+\frac{2 \pi}{\omega\left(h_{k}\right)}-\epsilon \frac{\partial S\left(t_{k+1}, h_{k}\right)}{\partial h_{k}} .
\end{gathered}
$$

The mapping (53) is implicit with respect to the variable $h_{k+1}$, while the mapping (55) is implicit in the variable $t_{k+1}$. One should note that only the phases $\Phi_{n}(t, h)$ of the generating function $S(t, h)$ depend on the energy $h$.

\section{MOTION IN A PERTURBED DOUBLE-WELL POTENTIAL}

As an example, we consider a motion of a particle in a double-well potential under external time-periodic perturbation. The system is described by the Hamiltonian

$$
\begin{gathered}
H=H_{0}(x, p)+\epsilon H_{1}(x, p, t), \\
H_{0}(x, p)=\frac{p^{2}}{2}-\frac{x^{2}}{2}+\frac{x^{4}}{4}, \\
\epsilon H_{1}(x, p, t)=\epsilon x \cos (\Omega t+\chi) .
\end{gathered}
$$

The potential function $U(x)=-x^{2} / 2+x^{4} / 4$ and the phase space of unperturbed motion $(\epsilon \equiv 0)$ are in Fig. 7. The unperturbed system has a single hyperbolic fixed point at $(x$ $=0, p=0)$ and two elliptic fixed points at $(x= \pm 1, p=0)$ [see Fig. 7(b)].

For $-1 / 4<H=H_{0}(x, p)<0$, a motion is trapped in potential wells (curves 1), and for $H>0$ a motion is untrapped (curve 3 ), and the separatrix $(H=0)$ is described by the curve 2. We introduce action-angle variables according to the definition given in Sec. III [Eq. (10)]. The action variable $I$ for the trapped motion $(H<0)$ is given by

$$
\begin{aligned}
I & =\frac{1}{2 \pi} \oint p(x ; H) d x \\
& =\frac{1}{\pi} \int_{-a}^{-b} \sqrt{2\left(H+x^{2} / 2-x^{4} / 4\right)} d x \\
& =\frac{2 a}{3 \pi}\left[E(k)-b^{2} K(k)\right],
\end{aligned}
$$




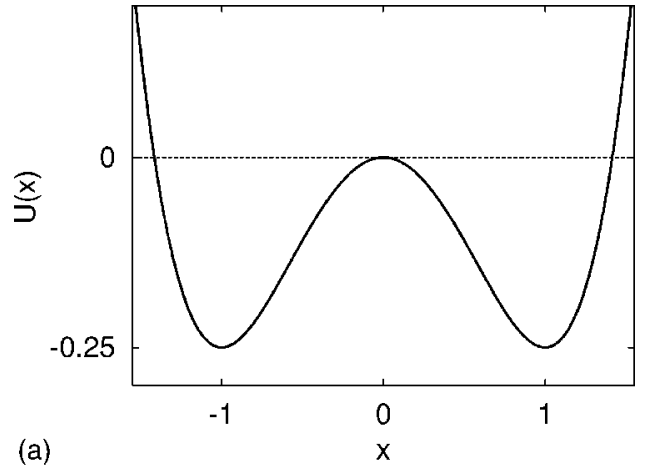

(a)

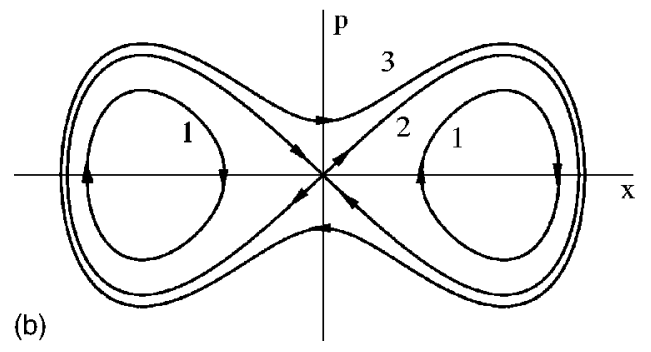

FIG. 7. (a) Double-well potential potential $U(x)=-x^{2} / 2+x^{4} / 4$; (b) phase space of motion.

where $(a, b)=\sqrt{1 \pm \sqrt{1+4 H}}$, and $K(k)$ and $E(k)$ are the complete elliptic integrals of the first kind and the second kind, respectively, with a module $k$,

$$
k=\frac{\sqrt{2}(1+4 H)^{1 / 4}}{(1+\sqrt{1+4 H})^{1 / 2}} .
$$

For the untrapped motion $(H>0)$ we introduce the action variable $I$ according to the definition given above [see Eq. (10)],

$$
\begin{aligned}
I & =\frac{1}{\pi} \int_{-a}^{0} \sqrt{2\left(H+x^{2} / 2-x^{4} / 4\right)} d x \\
& =\frac{\sqrt{2}}{3 \pi}\left[\left(a^{2}-b^{2}\right) E(k)+b^{2} K(k)\right],
\end{aligned}
$$

where

$$
k=\frac{\sqrt{1+\sqrt{1+4 H}}}{\sqrt{2}(1+4 H)^{1 / 4}} .
$$

The action $I(H)$ is a continuous function of energy $H$ at the separatrix $H=0$.

The unperturbed trajectory $x(t), p(t)$ can be determined by the second relation in Eq. (10). For the trapped motion we have

$$
\begin{gathered}
x(t)= \pm a \operatorname{dn}(u ; k), \\
u=\frac{a}{\sqrt{2}} \frac{\vartheta}{\omega(H)}=\frac{a}{\sqrt{2}}\left(t-t_{0}+\frac{\vartheta_{0}}{\omega(H)}\right),
\end{gathered}
$$

where $\operatorname{dn}(u ; k)$ is the Jacobi elliptic function. The solution (59) is chosen in order to have the orbit at the section $\Sigma_{c}(x$ $= \pm a$ ) when $\vartheta=0$. The quantity $\vartheta_{0}$ is the initial phase at the

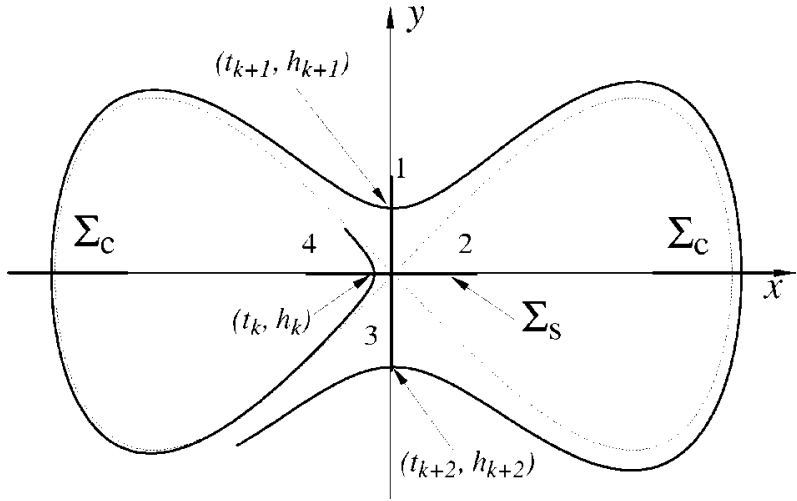

FIG. 8. Geometry of the separatrix mapping. The solid curve describes the perturbed orbit, and the dotted curve is the unperturbed separatrix.

time instant $t_{0}$. The unperturbed frequency, $\omega(H)$ $=d H_{0}(I) / d I$, of this motion is

$$
\omega(H)=\frac{\pi \sqrt{1+\sqrt{1+4 H}}}{\sqrt{2} K(k)} .
$$

Outside the potential wells $(H>0)$, the frequency is

$$
\omega(H)=\frac{\pi(1+4 H)^{1 / 4}}{K(k)} .
$$

Near the separatrix $(H \rightarrow 0)$ the periods $(60)$ and $(61)$ have the following asymptotics:

$$
\omega(H)=1 / \ln \frac{16}{|H|}+O(H) .
$$

The trajectory on the unperturbed separatrix $(H=0)$ is described by

$$
\begin{gathered}
x_{s}^{( \pm)}(t)= \pm \frac{\sqrt{2}}{\cosh \left(t-t_{c}\right)}, \\
p_{s}^{( \pm)}(t)=\mp \frac{\sqrt{2} \sinh \left(t-t_{c}\right)}{\cosh ^{2}\left(t-t_{c}\right)},
\end{gathered}
$$

where $t_{c}$ is a time instant when the orbit crosses the section $\Sigma_{c}$. The signs $( \pm)$ correspond to orbits in the right (left) half phase space, $(x>0)(x<0)$.

The geometry of the separatrix mapping for the Hamiltonian system (56) is shown in Fig. 8. The cross section $\Sigma_{c}$ consists of the segment of the $x$ axis located near the farthest crossing points of the unperturbed separatrix with the $x$ axis. The section $\Sigma_{s}$ is located near the saddle point $\left(x_{s}, p_{s}\right)$ and consists of two perpendicular segments of the $x$ and $p$ axes with the center at $\left(x_{s}=0, p_{s}=0\right)$. There are two types of saddle-saddle connections.

According to Eq. (56), the perturbation function $V_{n}(\mathcal{H}, \tau)$ is $x(\tau)$. According to the definition (38), the integrals $K$ $\equiv K_{n}$ are equal to $\pm K$, respectively, for the right $(x>0)$ and left $(x<0)$ halves of the phase space, where 


$$
\begin{aligned}
K & =\int_{-\infty}^{\infty} x_{s}^{(+)}(t) \cos (\Omega \tau) d \tau \\
& =\sqrt{2} \int_{-\infty}^{\infty} \frac{\cos (\Omega \tau) d \tau}{\cosh \tau} \\
& =\frac{\sqrt{2}}{\cosh (\pi \Omega / 2)}
\end{aligned}
$$

while $L_{n}=0$. Using the asymptotics of the frequency $\omega(H)$ (62) near the separatrix, one can write the two mappings, $\left(t_{k+1}, h_{k+1}\right)=\hat{M}^{( \pm)}\left(t_{k}, h_{k}\right)(42)$, corresponding to the two different saddle-saddle connections,

$$
\begin{gathered}
h_{k+1}=h_{k} \mp \epsilon \Omega K \sin \left(\varphi_{k}+\frac{\Omega}{2} \ln \frac{16}{\left|h_{k}\right|}+\chi\right), \\
\varphi_{k+1}=\varphi_{k}+\frac{\Omega}{2}\left(\ln \frac{16}{\left|h_{k}\right|}+\ln \frac{16}{\left|h_{k+1}\right|}\right),
\end{gathered}
$$

where the phase variable $\varphi=\Omega t$ is introduced. The map with the $(-)$ sign describes the right side of the phase space $(x>0)$, while the one with the $(+)$ sign corresponds to $(x<0)$.

The map (64) is an example of the algorithmic separatrix mapping, a term which is introduced in [20]. The dynamics of the system near the separatrix is described by the sequence of iterations of the maps $\hat{M}^{( \pm)}$. This sequence is determined by a certain rule. Let $S^{(+)}$and $S^{(-)}$be domains of phase space $(x, p)$ in the right $(x>0)$ and the left $(x<0)$ half planes, respectively. Then

$$
\hat{M}_{k+1}= \begin{cases}\hat{M}^{(+)} & \text {if } z_{k} \in S^{(+)} \text {and } h_{k}<0, \\ \hat{M}^{(-)} & \text {if } z_{k} \in S^{(+)} \text {and } h_{k}>0, \\ \hat{M}^{(+)} & \text {if } z_{k} \in S^{(-)} \text {and } h_{k}>0, \\ \hat{M}^{(-)} & \text {if } z_{k} \in S^{(-)} \text {and } h_{k}<0,\end{cases}
$$

where $z_{k}=\left(x_{k}, p_{k}\right)$. The separatrix mapping (64) has been applied in Ref. [46] to study the rescaling properties of Hamiltonian systems near the saddle points and the statistics of a residence time and a Poincaré recurrence.

\section{MAPPING FOR THE PERIODICALLY DRIVEN PENDULUM}

Consider the periodically driven pendulum given the following Hamiltonian:

$$
\begin{gathered}
H(q, p, t)=H_{0}(q, p)+\epsilon H_{1}(q, p, t), \\
H_{0}(q, p)=\frac{p^{2}}{2}-\omega_{0}^{2} \cos q, \\
\epsilon H_{1}(q, p, t)=\epsilon \omega_{0}^{2}[A \cos (q-\Omega t-\chi)+B \cos (q+\Omega t+\chi)] .
\end{gathered}
$$

The quantities $A$ and $B$ describe amplitudes of waves propagating in positive and negative directions of the $q$ axis.

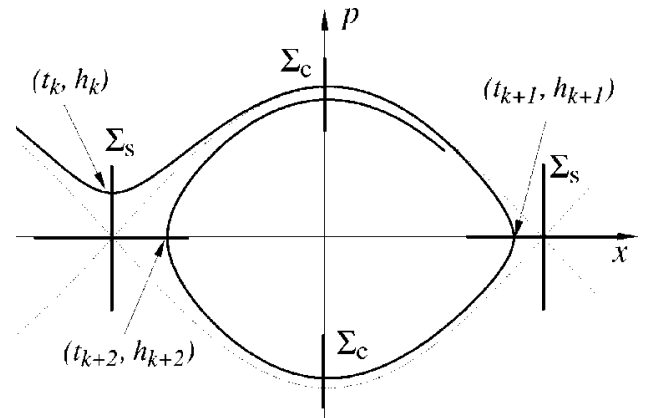

FIG. 9. Geometry of the separatrix mapping to the section $\Sigma_{s}$ for the periodically driven pendulum.

The geometry of the separatrix mapping $\left(t_{k}, h_{k}\right)$ $\rightarrow\left(t_{k+1}, h_{k+1}\right)$ is shown in Fig. 9. The sections $\Sigma_{s}$ on the $(x, p)$ plane consist of two perpendicular segments of $x$ and $p$ axes with the center at the hyperbolic fixed point $\left(x_{s}=2 \pi(s\right.$ $\left.+1 / 2), p_{s}=0\right)(s=0, \pm 1, \pm 2, \ldots)$. Sections $\Sigma_{c}$ consist of segments perpendicular to the unperturbed separatrices $\left(x_{s}(t), p_{s}(t)\right)$ at the midpoint between two consecutive saddle points, $x_{c}=2 \pi s$.

The system is described by the Hamiltonian (65). Changing the Hamiltonian to $H \rightarrow h=\left(H-\omega_{0}^{2}\right) / \omega_{0}^{2}, \omega_{0} t \rightarrow t, p / \omega_{0}$ $\rightarrow p$ we write the Hamiltonian in the form

$$
\begin{aligned}
H= & \frac{p^{2}}{2}-\cos x-1+\epsilon[A \cos (x-\Lambda t-\chi) \\
& +B \cos (x+\Lambda t+\chi)],
\end{aligned}
$$

where $\Lambda=\Omega / \omega_{0}$. The unperturbed motion $(\epsilon=0)$ is trapped for $H<0$ and untrapped for $H>0$ (curves 1 and 3 in Fig. 1). The action-angle variables $(I, \vartheta)$ for the unperturbed Hamiltonian (66) $(\epsilon=0)$ should be introduced in such a way that they should be continuous at the separatrix $H=0$. For this we define the action variable $I$ for the trapped motion as an integral taken along the segment of the orbit on the upper, $p>0$ (lower, $p<0$ ) half of the phase space $(x, p)$,

$$
\begin{aligned}
I & =\frac{1}{2 \pi} \int_{b}^{a} p(x, H) d x \\
& =\frac{1}{2 \pi} \sqrt{2(H+1+\cos x)} d x \\
& =\frac{4}{\pi}\left[E(k)-\left(1-k^{2}\right) K(k)\right],
\end{aligned}
$$

where $K(k), E(k)$ are the complete elliptic integrals with a module $k=\sqrt{1+H / 2}$, and $a, b$ are the roots of the equation $p(x, H)=0 \quad(2 \pi(s-1 / 2)<a, b<2 \pi(s+1 / 2))$. The corresponding angle variable $\vartheta$ is introduced as

$$
\begin{aligned}
\vartheta & =\frac{\partial}{\partial I} \int_{2 \pi s}^{x} p(x, H) d x \\
& =\omega(H) \int_{0}^{x} \frac{d x}{\sqrt{2(H+1+\cos x)}} \\
& =\omega(H) F\left(k^{-1} \arcsin (x / 2) ; k\right),
\end{aligned}
$$


with the conditions that $\vartheta=0$ at the sections $\Sigma_{c}(x=2 \pi s)$ and $\vartheta(\bmod 2 \pi)= \pm \pi$ at $\Sigma_{s}[x=2 \pi(s+1 / 2)]$. From Eq. (68) it follows that

$$
x(\vartheta ; H)=2 \arcsin [k \operatorname{sn}(\vartheta / \omega(H) ; k)] .
$$

The frequency of motion $\omega(H)=d H(I) / d I=2 \pi / K(k)$ has the following asymptotics near the separatrix:

$$
\omega(H)=\frac{2 \pi}{\ln (32 /|H|)} \text { for } H \rightarrow-0 .
$$

For the untrapped motion $(H>0)$, the action-angle variables are introduced as

$$
\begin{gathered}
I=\frac{1}{2 \pi} \int_{-2 \pi(s-1 / 2)}^{2 \pi(s+1 / 2)} p(x, H) d x=\frac{4}{\pi k} E\left(k^{-1}\right), \\
\vartheta=\frac{\partial}{\partial I} \int_{2 \pi s}^{x} p\left(x^{\prime}, H\right) d x^{\prime}=\omega(H) k^{-1} F\left(x / 2, k^{-1}\right), \\
\sin (x / 2)=\operatorname{sn}\left(k \vartheta / \omega(H) ; k^{-1}\right)=\operatorname{sn}\left(k\left(t-t_{0}\right) ; k^{-1}\right) .
\end{gathered}
$$

The frequency $\omega(H)=2 \pi k / K\left(k^{-1}\right)$ has the same asymptotics (69) at $H \rightarrow+0$.

The orbits on the upper (lower) branches of the separatrix $(H=0)$ are

$$
\begin{aligned}
\sin x^{ \pm}(t) & = \pm \frac{\sinh \left(t-t_{c}\right)}{\cosh ^{2}\left(t-t_{c}\right)}, \\
\cos x^{ \pm}(t) & =\frac{2}{\cosh ^{2}\left(t-t_{c}\right)}-1,
\end{aligned}
$$

where the sign $(+)$ corresponds to the orbit along the upper separatrix $(p>0)$, and the sign (-) to the one on the lower separatrix $(p<0)$.

The perturbed Hamiltonian $\mathcal{H}_{1}\left(t, \vartheta, p_{0}\right)$ in Eq. (13),

$$
\begin{aligned}
\mathcal{H}_{1}\left(t, \vartheta, p_{0}\right)= & \epsilon\left\{A \cos \left[x\left(\vartheta, p_{0}\right)-\Lambda t-\chi\right]+B \cos \left[x\left(\vartheta, p_{0}\right)\right.\right. \\
& +\Lambda t+\chi]\},
\end{aligned}
$$

can be presented in the form (25) with

$$
\begin{gathered}
V_{1}\left(\mathcal{H}, t-t_{c}\right)=(A+B) \cos x(\vartheta ; H), \\
\Omega_{1}=\Lambda, \quad \chi_{1}=\chi, \\
V_{2}\left(\mathcal{H}, t-t_{c}\right)=(A-B) \sin x(\vartheta ; \mathcal{H}), \\
\Omega_{2}=\Lambda, \quad \chi_{2}=\chi-\frac{\pi}{2} .
\end{gathered}
$$

Remember that $V_{n}\left(\mathcal{H}, t-t_{c}\right) \equiv H_{n}(\mathcal{H}, \vartheta)$. Using Eq. (70), we have on the separatrix

$$
\begin{gathered}
V_{1}(0, \tau)=(A+B)\left(\frac{2}{\cosh ^{2} \tau}-1\right), \\
V_{2}(0, \tau)= \pm(A-B) \frac{\sinh \tau}{\cosh ^{2} \tau} .
\end{gathered}
$$

\section{A. Mapping to sections $\mathbf{\Sigma}_{s}$}

First we construct the separatrix mapping at the sections $\Sigma_{s}$ using the general formula (42). Using Eq. (38), one obtains that $K_{2}=L_{1}=0$ and

$$
\begin{gathered}
K_{1}=2(A+B) \int_{-\infty}^{\infty} \frac{\cos (\Lambda \tau) d t}{\cosh ^{2} \tau}=\frac{2 \pi \Lambda(A+B)}{\sinh (\pi \Lambda / 2)}, \\
L_{2}= \pm(A-B) \int_{-\infty}^{\infty} \frac{\sinh \tau \sin (\Lambda \tau) d t}{\cosh ^{2} \tau}= \pm \frac{2 \pi \Lambda(A-B)}{\cosh (\pi \Lambda / 2)} .
\end{gathered}
$$

Inserting these coefficients into the separatrix mapping (42) and using the asymptotics of $\omega(H)(69)$, we obtain

$$
\begin{gathered}
h_{k+1}=h_{k}-\epsilon K^{ \pm} \sin \left(\varphi_{k}+\frac{\Lambda}{2} \ln \frac{32}{\left|h_{k}\right|}+\chi\right), \\
\varphi_{k+1}=\varphi_{k}+\frac{\Lambda}{2}\left(\ln \frac{32}{\left|h_{k+1}\right|}+\ln \frac{32}{\left|h_{k}\right|}\right),
\end{gathered}
$$

where $\varphi=\Lambda t$, and

$$
K^{ \pm}=\Lambda\left(K_{1}+L_{2}\right)=\frac{4 \pi \Lambda^{2}}{\sinh (\pi \Lambda)}\left[A e^{ \pm \pi \Lambda / 2}+B e^{\mp \pi \Lambda / 2}\right] .
$$

The sign $( \pm)$ corresponds to the integral taken along the separatrix on the upper (lower) half phase space, $p>0$ $(p<0)$, respectively. The dynamics of the system is fully determined by two mappings (72).

Let $\left(\varphi_{k}, h_{k}\right)$ be the phase and the energy at the $k$ th mapping step. Suppose also that $\left(x_{k}, p_{k}\right)$ are the corresponding phase-space coordinates. The sequence of the mapping iteration $\hat{M}_{k}:\left(\varphi_{k+1}, h_{k+1}\right)=\hat{M}_{k+1}\left(\varphi_{k}, h_{k}\right)$ and the coordinates $\left(x_{k+1}, p_{k+1}\right)$ after one map iteration are determined by the following algorithm:

$$
\hat{M}_{k+1}= \begin{cases}\hat{M}^{(+)} & \text {if } \hat{M}_{k}=\hat{M}^{(+)} \text {and } h_{k}>0, \\ \hat{M}^{(-)} & \text {if } \hat{M}_{k}=\hat{M}^{(-)} \text {and } h_{k}>0, \\ \hat{M}^{(-)} & \text {if } \hat{M}_{k}=\hat{M}^{(+)} \text {and } h_{k}<0, \\ \hat{M}^{(+)} & \text {if } \hat{M}_{k}=\hat{M}^{(-)} \text {and } h_{k}<0,\end{cases}
$$

where $\hat{M}^{( \pm)}$are mappings (72) along the upper and lower branches, respectively.

The separatrix mapping (72) has been used in Ref. [46] to analyze the rescaling properties of the Hamiltonian system near the hyperbolic saddle point and to study a chaotic transport along the stochastic layer.

\section{B. Mapping to sections $\boldsymbol{\Sigma}_{c}$}

For the problem under consideration, there are two different types of sections $\Sigma_{c}$, namely $\Sigma_{c}^{+}$and $\Sigma_{c}^{-}$, corresponding to the upper and lower branches of the separatrix. The geometry of the separatrix mapping to the cross sections $\Sigma_{c}^{ \pm}$is schematically shown in Fig. 10. In general, there are four independent mappings of the sections $\Sigma_{c}^{ \pm}$to $\Sigma_{c}^{ \pm}$which fully de- 


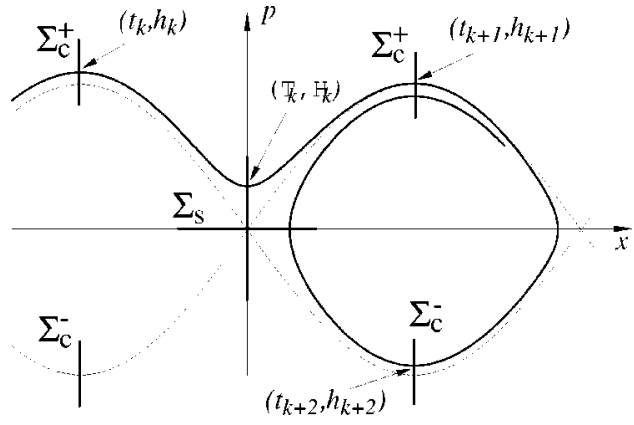

FIG. 10. Geometry of the separatrix mapping to the sections $\Sigma_{c}^{ \pm}$ for the periodically driven pendulum.

termine the dynamics of the system. These mapping should be constructed in two steps: in the first step, one should find the map from the section $\Sigma_{c}^{ \pm}$to $\Sigma_{s}$ along a certain saddlesaddle connection, and in the second step one should map $\Sigma_{s}$ to $\Sigma_{c}^{ \pm}$along another saddle-saddle connection which depends on the sign energy on the section $\Sigma_{s}$. Therefore, the dynamics of the system is then fully determined, in general, by four independent mappings, $\hat{T}_{1}^{( \pm)}$and $\hat{T}_{2}^{( \pm)}$, where $\hat{T}_{1}^{( \pm)}$stands for the mapping of variables $\left(t_{k}, h_{k}\right) \in \Sigma_{c}^{ \pm}$to $\left(\mathcal{T}_{k}, \mathcal{H}_{k}\right) \in \Sigma_{s}$ along upper $(+)$ or lower $(-)$ branches of the separatrix, and $\hat{T}_{2}^{( \pm)}$ stands for the mapping of variables $\left(\mathcal{T}_{k}, \mathcal{H}_{k}\right) \in \Sigma_{s}$ to $\left(t_{k+1}, h_{k+1}\right) \in \Sigma_{c}^{ \pm}$along upper $(+)$or lower $(-)$branches of the separatrix, respectively.

These mappings can be constructed using a general method described in Sec. III. Below, we present nonsymmetric forms of these mappings similar to ones (33), (36) obtained in Sec. III F. Using Eqs. (16) and (26), one can show that the mapping $\left(\mathcal{T}_{k}, \mathcal{H}_{k}\right)=\hat{T}_{1}^{( \pm)}\left(t_{k}, h_{k}\right)$ is given by

$$
\begin{gathered}
\mathcal{H}_{k}=h_{k}-\frac{1}{2} \epsilon\left[K^{ \pm} \sin \left(\varphi_{k}+\chi\right)+L^{ \pm} \cos \left(\varphi_{k}+\chi\right)\right], \\
\Phi_{k}=\varphi_{k}+\frac{\Lambda}{2} \ln \frac{32}{\left|\mathcal{H}_{k}\right|}, \Phi_{k}=\Omega \mathcal{T}_{k},
\end{gathered}
$$

and $\left(t_{k+1}, h_{k+1}\right)=\hat{T}_{2}^{( \pm)}\left(\mathcal{T}_{k}, \mathcal{H}_{k}\right)$ is

$$
\begin{gathered}
\varphi_{k+1}=\Phi_{k}+\frac{\Lambda}{2} \ln \frac{32}{\left|\mathcal{H}_{k}\right|}, \\
h_{k+1}=\mathcal{H}_{k}-\frac{1}{2} \epsilon\left[K^{ \pm} \sin \left(\varphi_{k+1}+\chi\right)-L^{ \pm} \cos \left(\varphi_{k+1}+\chi\right)\right] .
\end{gathered}
$$

In Eqs. (75) and (76) the coefficient $K^{ \pm}$is determined by Eq. (73), and

$$
\begin{gathered}
L^{ \pm}=2 \Lambda[A(S \mp C)+B(S \pm C)], \\
S=2 \int_{0}^{\infty} \frac{\sin (\Lambda t) d t}{\cosh ^{2} t}, \quad C=\int_{0}^{\infty} \frac{\cos (\Lambda t) \sinh t d t}{\cosh ^{2} t} .
\end{gathered}
$$

According to the scheme shown in Fig. 10, the mapping

$$
\left(t_{k+1}, h_{k+1}\right)=\hat{M}\left(t_{k}, h_{k}\right)
$$

of $\Sigma_{c}^{ \pm}$to $\Sigma_{c}^{ \pm}$is presented by two consecutive mappings $\hat{T}_{1}^{ \pm}$, $\hat{T}_{2}^{ \pm}$, given by the following rules:

$$
\hat{M}= \begin{cases}\hat{T}_{2}^{+} \hat{T}_{1}^{+} \quad \text { if } \quad\left(t_{k}, h_{k}\right) \in \Sigma_{c}^{+} \text {and } \mathcal{H}_{k}>0, \\ \hat{T}_{2}^{+} \hat{T}_{1}^{+} \text {if } \quad\left(t_{k}, h_{k}\right) \in \Sigma_{c}^{+} \text {and } \mathcal{H}_{k}<0, \\ \hat{T}_{2}^{+} \hat{T}_{1}^{-} \text {if } \quad\left(t_{k}, h_{k}\right) \in \Sigma_{c}^{-} \text {and } \mathcal{H}_{k}<0, \\ \hat{T}_{2}^{-} \hat{T}_{1}^{-} \text {if } \quad\left(t_{k}, h_{k}\right) \in \Sigma_{c}^{-} \text {and } \mathcal{H}_{k}>0 .\end{cases}
$$

Consider a particular case $A=B=1$ when the problem coincides with the one considered in Sec. II. Then the coefficient $K^{ \pm}=W / \epsilon$ [see Eq. (7)], $L^{ \pm}=L=\Lambda S$. The mapping (77) can be written in the following simplified form as a mapping $\left(t_{k}, \mathcal{H}_{k-1}\right) \rightarrow\left(t_{k+1}, \mathcal{H}_{k}\right)$ for the noncanonical variables $(t, \mathcal{H})$,

$$
\begin{gathered}
\mathcal{H}_{k}=\mathcal{H}_{k-1}-W \sin \left(\varphi_{k}+\chi\right), \\
\varphi_{k+1}=\varphi_{k}+\Lambda \ln \frac{32}{\left|\mathcal{H}_{k}\right|},
\end{gathered}
$$

with the energy $\mathcal{H}_{k}$ defined at the section $\Sigma_{s}$ and time (or phase), and $\varphi_{k}$ defined at the sections $\Sigma_{c}^{ \pm}$. The mapping (79) formally coincides with the conventional separatrix mapping (8) (supposing $\chi=0$ ). The latter can also be obtained from the mapping (72) by replacement of the phase, $\varphi_{k}$ $+\Lambda / 2 \ln \left(32 /\left|h_{k}\right|\right) \rightarrow \varphi_{k}$. The mapping (79) clarifies the meaning of variables $(t, h)$ in the conventional separatrix mapping (8).

In spite of this coincidence, however, there is a fundamental difference between these mappings. The canonical mappings (72), (77) are supplemented with the corresponding rules (74), (78) of their application which fully determine the evolution of the system in phase space. The formal derivation of the conventional separatrix mapping (8) by calculating the increments does not give any rules to apply this mapping to study the dynamics of the system. For this reason, it has been mostly employed to estimate the width of the stochastic layer.

\section{MAPPING FOR THE PERIODICALLY DRIVEN MORSE OSCILLATOR}

Consider the example of a Hamiltonian system with the saddle point located at infinity, namely the classical Morse oscillator driven by time-periodic force. This system has been widely used as the main model in the studies of stochastic excitation and dissociation of diatomic molecules in a microwave field and associated with the onset of chaos (see Refs. [54,55]). The model is described by the Hamiltonian

$$
H=\frac{p^{2}}{2 m}+D\left(1-e^{-x / a}\right)^{2}+x E_{0} d \cos (\Omega t+\chi),
$$

where $D$ is the depth of the potential well, $d$ is a molecule's dipole moment, $\Omega$ and $E_{0}$ are the frequency and the amplitude of a microwave field, and the parameter $a$ is the effective width of the unperturbed potential function $U(x)$ 

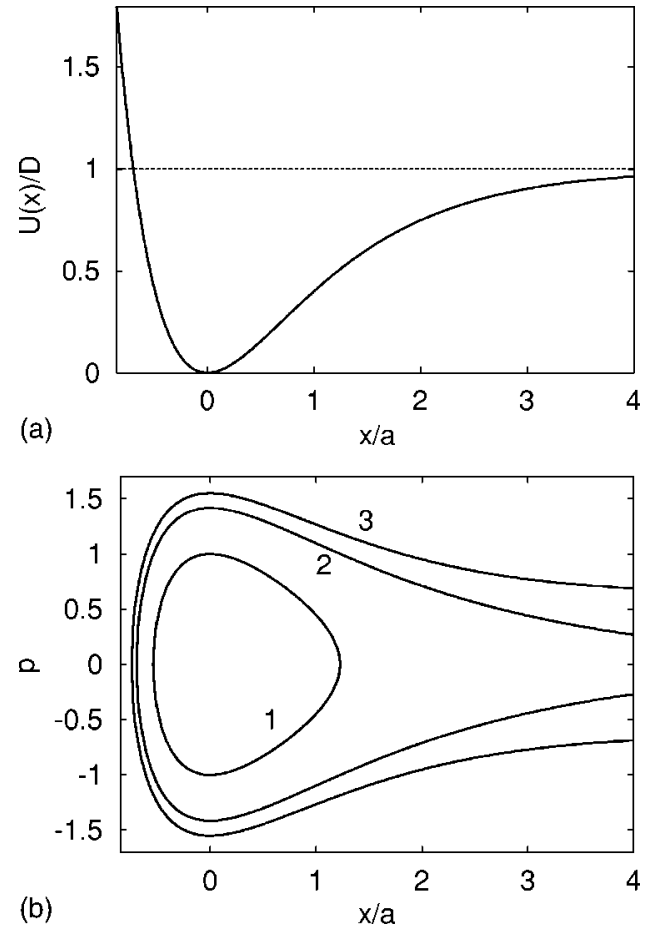

FIG. 11. (a) Morse potential $U(x)=D\left(1-e^{-x / a}\right)^{2}$; (b) phase space of the unperturbed Hamiltonian (80) $\left(E_{0}=0\right)$ : curve 1 corresponds to the trapped motion $(H<1)$, curve 2 to the separatrix $(H=1)$, and curve 3 to the unbounded motion $(H>1)$.

$=D\left(1-e^{-x / a}\right)^{2}$, shown in Fig. 11. The latter has a minimum at $x=0$.

The phase-space structure of unperturbed motion $\left(E_{0}=0\right)$ is shown in Fig. 11(b). The unperturbed motion is trapped when $H<D$ (curve 1), and it is unbounded when $H>D$ (curve 3). There are two fixed points of unperturbed motion: the elliptic fixed point at $(x=0, p=0)$ and the nonhyperbolic saddle point at $(x=\infty, p=0)$. The oscillation frequency near the fixed point $(x=0, p=0)$ is $\omega_{0}=(2 D / m)^{1 / 2} a^{-1}$.

Introducing the normalized energy, $h=H / D-1$, one can show that the unperturbed orbit of trapped motion, $h<0$, is described by

$$
\begin{gathered}
\exp (x / a)=|h|^{-1}[1-\sqrt{1-|h|} \cos \vartheta], \\
p=\frac{m a \omega(h) \sqrt{1-|h|} \sin \vartheta}{1-\sqrt{1-|h|} \cos \vartheta},
\end{gathered}
$$

where $\vartheta=\vartheta_{0}+\omega(h)\left(t-t_{c}\right)$ is the angle variable, $t_{c}$ is a time instant when the orbit crosses the point $\left(x_{1}, p=0\right)$, and $x_{1}$ is a left turning point of motion $\left[p\left(x_{1}\right)=0\right]$. We set $\vartheta_{0}=0$ in order to have $\vartheta\left(t_{c}\right)=0$. The relation between the action variable $(I)$ and the energy $h$ is given by

$$
I=\frac{1}{2 \pi} \oint p d x=\frac{1}{\pi} \int_{x_{1}}^{x_{2}} p d x=a \sqrt{2 m D}\left(1-|h|^{1 / 2}\right),
$$

$$
H=D\left[1-\left(1-I / I_{0}\right)^{2}\right],
$$

where $I_{0}=a \sqrt{2 m D}$, and $x_{1}, x_{2}$ are two turning points of motion,

$$
x_{1,2}=a \ln [(1 \mp \sqrt{1-|h|}) / h \mid], \quad x_{1}<x_{2} .
$$

The frequency of oscillations $\omega(h)=d H(I) / d I$ is

$$
\omega(h)=\omega_{o}|h|^{1 / 2} .
$$

According to quasiclassical quantization rules $I=\hbar(m$ $+1 / 2)(m=0,1,2, \ldots)$, where $\hbar$ is Planck's constant, one can obtain the discrete energetic levels of the Morse oscillator,

$$
H_{m}=D\left\{1-\left[1-\hbar(m+1 / 2) / I_{0}\right]^{2}\right\} .
$$

For the lowest energetic levels, $m \hbar \ll I_{0}$, we have the energy levels of the harmonic oscillator: $H_{m} \approx \hbar \omega_{0}(m+1 / 2)$.

The motion on the separatrix $h=0$ is given by the formula

$$
\begin{aligned}
& x_{s}(t)=a \ln \frac{1+\omega_{0}^{2}\left(t-t_{c}\right)^{2}}{2}, \\
& p_{s}(t)=2 p_{0} \frac{\omega_{0}\left(t-t_{c}\right)}{1+\omega_{0}^{2}\left(t-t_{c}\right)^{2}},
\end{aligned}
$$

where $p_{0}=m a \omega_{0}=\sqrt{2 D m}$. The orbits of unbounded motion, $h>0$, are given by

$$
\exp (x / a)=h^{-1}\left\{-1+\sqrt{1+h} \cosh \left[\omega(h)\left(t-t_{c}\right)\right]\right\} .
$$

\section{A. Mappings}

We formulate the Hamiltonian system (80) in the extended phase space of the action-angle $(I, \vartheta)$ and the timeenergy $\left(t, p_{0}\right)$ variables in the form (12) with the Hamiltonian (13),

$$
\begin{gathered}
H_{0}(I)=-\left(1-I / I_{0}\right)^{2}, \\
\epsilon H_{1}\left(t, \vartheta, p_{0}\right)=\epsilon \frac{x\left(\vartheta, p_{0}\right)}{a} \cos (\Omega t+\chi),
\end{gathered}
$$

where $\epsilon=E_{0} a d / D$ is the dimensionless perturbation parameter. We intend to construct the Poincaré return map $\left(t_{k}, h_{k}\right)$ $\rightarrow\left(t_{k+1}, h_{k+1}\right)$ near the separatrix to the cross sections $\Sigma_{s}$ and $\Sigma_{c}$ on the phase space. The geometry of this mapping is plotted in Fig. 12. The cross sections $\Sigma_{c}$ and $\Sigma_{s}$ consist of the segments on the $x$ axis covering the left, $x_{1}$, and the right, $x_{2}$, turning points of unperturbed motion, respectively. The general form of the corresponding mapping in the first order of $\epsilon$ is given by Eq. (23) with the generating function (21). Nonsymmetric forms of the mappings are given by Eq. (33) or Eq. (36) with the generating function (34).

The stochastic layer formed near the separatrix of the Morse oscillator is sufficiently large even for small perturbations. The variation of energy in the stochastic layer may be large enough that deviation of the generating function $S(H, t)$ (34), (31) from its value $S(H=0, t)$ at the unperturbed separatrix $H=0$ would not be negligible. In this case, the depen- 


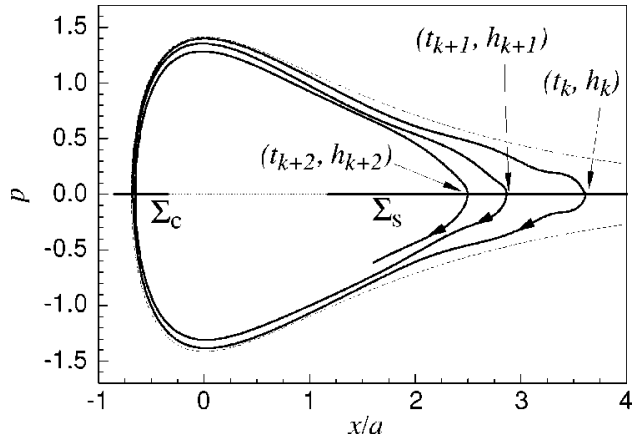

FIG. 12. Geometry of the separatrix mapping to the section $\Sigma_{s}$ for the periodically driven Morse oscillator. A dashed curve describes unperturbed separatrix.

dence of the generating function $S(H, t)$ on the energy variable $H$ becomes important.

\section{The symmetric mapping}

According to the relations (84), (81), the perturbation function $V_{n}(\mathcal{H}, \tau)$ is equal to $V_{n}=x(\vartheta, H) / a$. Using Eq. (82) for the frequency $\omega(h)$, the integrals (31) can be reduced to

$$
\begin{aligned}
R^{ \pm}(h) & =\frac{1}{a} \int_{\mp \pi / \omega(h)}^{0} x(\tau, h) e^{i \Omega \tau} d \tau=\frac{1}{i \Omega a} \int_{0}^{\mp \pi / \omega(h)} e^{i \Omega \tau} \frac{d x}{d t} d \tau \\
& =\frac{\sqrt{1-|h|}}{i \Omega} \int_{0}^{\mp \pi} \frac{e^{i \Lambda|h|^{-1 / 2} \eta} \sin \eta}{1-\sqrt{1-|h|} \cos \eta} d \eta,
\end{aligned}
$$

where $\Lambda=\Omega / \omega_{0}$. Then according to Eq. (28), the generating function takes the form

$$
\begin{aligned}
S\left(t_{k} \pm 0, \mathcal{H}\right)= & K^{ \pm}(\mathcal{H}) \Omega^{-1} \cos \left(\Omega t_{k} \pm \pi \Lambda|\mathcal{H}|^{-1 / 2}+\chi\right) \\
& -L^{ \pm}(\mathcal{H}) \Omega^{-1} \sin \left(\Omega t_{k} \pm \pi \Lambda|\mathcal{H}|^{-1 / 2}+\chi\right),
\end{aligned}
$$

where $K^{+}(h)=-K^{-}(h)=-K(h), L^{+}(h)=L^{-}(h)=-L(h)$, and

$$
\begin{aligned}
& K(h)=\sqrt{1-|h|} \int_{0}^{\pi} \frac{\sin \tau \sin \left(\Lambda \tau /|h|^{1 / 2}\right)}{1-\sqrt{1-|h|} \cos \tau} d \tau, \\
& L(h)=\sqrt{1-|h|} \int_{0}^{\pi} \frac{\sin \tau \cos \left(\Lambda \tau /|h|^{1 / 2}\right)}{1-\sqrt{1-|h|} \cos \tau} d \tau .
\end{aligned}
$$

The asymptotic estimation of the integral $K(h)$ is given in Appendix. A. The dependence of the integral $K(h)$ on $h$ is shown in Fig. 13 at a fixed value of the parameter $\lambda=2$.

Using the generating function (86), the symmetric mapping (23) can be written as

$$
\begin{gathered}
\mathcal{H}=h_{k}+\epsilon\left[K(\mathcal{H}) \sin \alpha_{k}+L(\mathcal{H}) \cos \alpha_{k}\right], \\
\Phi_{k}=\Omega t_{k}+\epsilon\left(\frac{d K(\mathcal{H})}{d \mathcal{H}} \cos \alpha_{k}-\frac{d L(\mathcal{H})}{d \mathcal{H}} \sin \alpha_{k}\right) \\
+\epsilon \frac{\pi \Lambda}{2|H|^{3 / 2}}\left[K(\mathcal{H}) \sin \alpha_{k}+L(\mathcal{H}) \cos \alpha_{k}\right],
\end{gathered}
$$
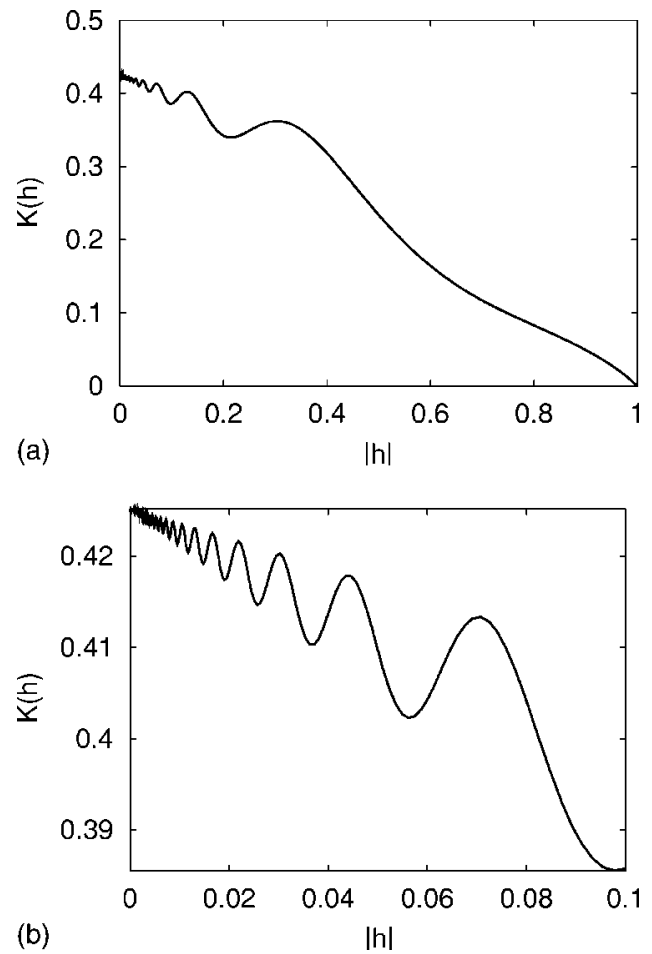

FIG. 13. Dependence of the integral $K(h)$ on $|h|$ : (a) in the whole interval of $h$; (b) in the interval of small $h$. Parameter $\lambda=2$.

$$
\begin{gathered}
h_{k+1}=\mathcal{H}+\epsilon\left[K(\mathcal{H}) \sin \alpha_{k+1}-L(\mathcal{H}) \cos \alpha_{k+1}\right], \\
\Omega t_{k+1}=\Phi_{k}+\frac{2 \pi \Lambda}{|\mathcal{H}|^{1 / 2}}+\epsilon\left(\frac{d K(\mathcal{H})}{d \mathcal{H}} \cos \alpha_{k+1}+\frac{d L(\mathcal{H})}{d \mathcal{H}} \sin \alpha_{k+1}\right) \\
-\epsilon \frac{\pi \Lambda}{2|\mathcal{H}|^{3 / 2}}\left[K(\mathcal{H}) \sin \alpha_{k+1}-L(\mathcal{H}) \cos \alpha_{k+1}\right],
\end{gathered}
$$

where

$$
\alpha_{k}=\Omega t_{k}+\frac{\pi \Lambda}{|\mathcal{H}|^{1 / 2}}+\chi, \alpha_{k+1}=\Omega t_{k+1}-\frac{\pi \Lambda}{|\mathcal{H}|^{1 / 2}}+\chi .
$$

The first set of equations (88) is implicit with respect to the energy variable $\mathcal{H}$, and the second set (89) is implicit with respect to the time $t_{k+1}$.

The mapping (88), (89) can be simplified using a smallness of the perturbation parameter $\epsilon$. Carrying out the transformations similar to ones made in Sec. III G and neglecting the terms of order $\epsilon^{2}$, one obtains

$$
\begin{aligned}
h_{k+1}= & h_{k}-2 \epsilon K\left(h_{k+1}\right) \sin \left(\varphi_{k}+\frac{\pi \Lambda}{\left|h_{k}\right|^{1 / 2}}+\chi\right), \\
\varphi_{k+1}= & \varphi_{k}+\frac{\pi \Lambda}{\left|h_{k}\right|^{1 / 2}}+\frac{\pi \Lambda}{\left|h_{k+1}\right|^{1 / 2}} \\
& -2 \epsilon \frac{d K\left(h_{k+1}\right)}{d h_{k+1}} \cos \left(\varphi_{k}+\frac{\pi \Lambda}{\left|h_{k}\right|^{1 / 2}}+\chi\right) .
\end{aligned}
$$

One can show that $\left|\partial\left(h_{k+1}, t_{k+1}\right) / \partial\left(h_{k}, t_{k}\right)\right|=1$, i.e., the map- 
ping (90) is area-preserving. It is also invariant with respect to the time reversing: $k \leftrightarrow k+1$.

\section{Nonsymmetric mappings}

We present also the nonsymmetric form of the separatrix mapping. Particularly, we consider the mapping in the form (33). Putting $\vartheta=-\pi$ in Eq. (31), one can show that $L(h)=0$ and $K(h)$ is determined by Eq. (87). Then the generating function (34) becomes

$$
S\left(t_{k}, h_{k+1}\right)=-2 K\left(h_{k+1}\right) \Omega^{-1} \cos \left(\Omega t_{k}+\frac{\pi \Lambda}{\left|h_{k+1}\right|^{1 / 2}}+\chi\right) .
$$

Then the separatrix mapping (33) takes the following form:

$$
\begin{gathered}
h_{k+1}=h_{k}-2 \epsilon K\left(h_{k+1}\right) \sin \left(\varphi_{k}+\frac{\pi \Lambda}{\left|h_{k+1}\right|^{1 / 2}}+\chi\right) \\
\varphi_{k+1}=\varphi_{k}+\frac{2 \pi \Lambda}{\left|h_{k+1}\right|^{1 / 2}}-2 \epsilon \frac{d K\left(h_{k+1}\right)}{d h_{k+1}} \cos \left(\varphi_{k}+\frac{\pi \Lambda}{\left|h_{k+1}\right|^{1 / 2}}+\chi\right) \\
-\frac{\pi \Lambda}{\left|h_{k+1}\right|^{3 / 2}} \epsilon K\left(h_{k+1}\right) \sin \left(\varphi_{k}+\frac{\pi \Lambda}{\left|h_{k+1}\right|^{1 / 2}}+\chi\right) .
\end{gathered}
$$

This map can also be transformed into the form (90) by eliminating the last term in the second Eq. (91) using the first equation, and neglecting the terms of order $\epsilon^{2}$.

We have compared the obtained mapping with the numerical integration of continuous system (80). The phase space of the separatrix mapping (14) near the separatrix region is plotted in Fig. 14(a) for the perturbation parameter $\epsilon=0.01$ and the normalized frequency $\Lambda=\Omega / \omega_{0}=4$. It is supposed that the orbit leaves the system when the energy $h$ exceeds zero, $h>0$, which corresponds to the unbounded motion. The phase space of the separatrix mapping has been compared with the ones obtained by the direct numerical integration of system (80) using the symplectic integrator scheme proposed in Ref. [58]. The results are shown in Fig. 14(b). As seen from Figs. 14(a) and 14(b), the separatrix mapping reproduces quantitatively well all features of the regular and chaotic motion of the system, namely the locations of KAM islands and their widths.

\section{THE KEPLER MAP}

In this section, we construct a mapping for another Hamiltonian system with a saddle point located at infinity, namely for a one-dimensional hydrogen atom in the field of a monochromatic electromagnetic wave. The latter problem has been the subject of numerous studies related to the chaotic ionization of a highly excited hydrogen atom in a microwave field (see the review [56]). This problem is similar to the above-mentioned problem of dissociation of molecules in a microwave field. In several publications [22-25,27], the socalled Kepler map has been introduced to study this problem. However, the variables of this map are not canonical. Below, we derive the Kepler map in canonical variables using the method described in the previous sections. It has a form similar to the map (90) for the driven Morse oscillator, and
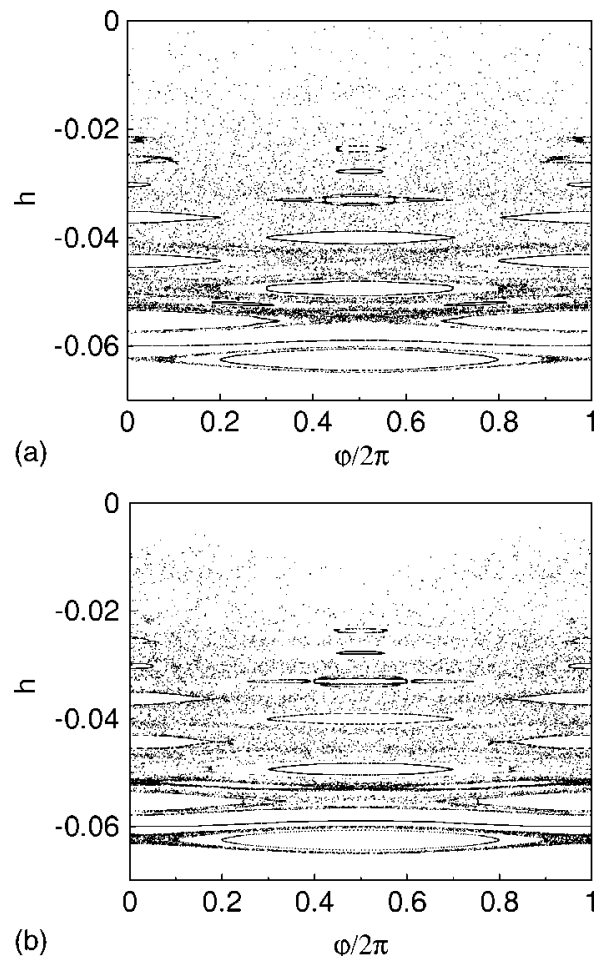

FIG. 14. (a) Phase space of the separatrix mapping (90); (b) a corresponding section obtained by a direct numerical integration of Hamiltonian system (80). Perturbation parameter $\epsilon=0.01$, normalized frequency $\Lambda=4$.

describes the classical motion of an electron in a onedimensional model of the hydrogen atom in a monochromatic electromagnetic field.

In atomic units $\left(m_{e}=\hbar=e=1\right)$, this model is described by the Hamiltonian

$$
H=H(x, p, t)=\frac{p^{2}}{2}-\frac{1}{x}+x F \cos (\Omega t+\chi), \quad x \geqslant 0,
$$

where $\Omega$ and $F$ are the microwave frequency and amplitude, respectively. In the absence of a microwave field $(F=0)$, the classical orbit $x\left(t-t_{0}, H\right)$ of a bounded electron $(H<0)$ is given by

$$
\vartheta=\omega(H)\left(t-t_{0}\right)=\arcsin \sqrt{\frac{x}{x_{c}}}-\sqrt{\frac{x}{x_{c}}\left(1-\frac{x}{x_{c}}\right)},
$$

where $t_{0}$ is the moment of time when an electron reflects from the boundary $x=0$ (perihelion), and $x_{c}=1 /|H|$ is the turning point of classical motion (aphelion). The frequency of motion $\omega(H)$ is determined by the relation between the action $(I)$ and energy $(H)$,

$$
\omega(H)=\frac{d H_{0}(I)}{d I}=\frac{1}{I^{3}}=(2|H|)^{3 / 2},
$$




$$
H_{0}(I)=-\frac{1}{2 I^{2}}
$$

From the perturbed Hamiltonian $\epsilon H_{1}=x F \cos (\Omega t+\chi)$ in Eq. (92) it follows that $V_{n}(H, \tau=x(\tau, H)$, and using the relation (93) the integral $K_{n}(H)$ (35) can be reduced to

$$
\begin{aligned}
K_{n}(H) & =\int_{-\pi / \omega(H)}^{\pi / \omega(H)} x(\tau, H) \cos \Omega \tau d \tau \\
& =-2 \frac{1}{\Omega} \int_{0}^{x_{c}} \sin \Omega \tau(x) d x \\
& =-\frac{2 \pi}{|H| \Omega} \mathbf{J}_{\nu}^{\prime}(\nu),
\end{aligned}
$$

$L_{n}(H)=0$, where $\nu=\Omega / \omega(H)$, and $\mathbf{J}_{\nu}^{\prime}(z) \equiv d \mathbf{J}_{\nu}(z) / d z$ is the derivative of the Auger function

$$
\mathbf{J}_{\nu}(z)=\frac{1}{\pi} \int_{0}^{\pi} \cos (\nu x-z \sin x) d x .
$$

For $\nu \gg 1$ (or $|H| \ll \Omega^{3 / 2} / 2$ ), it has the following asymptotics [57]:

$$
\mathbf{J}_{\nu}^{\prime}(\nu) \approx \frac{a}{\nu^{2 / 3}}-\frac{b}{\nu^{4 / 3}}-\frac{\sin \nu \pi}{4 \pi \nu^{2}}
$$

where

$$
a=\frac{2^{2 / 3}}{3^{1 / 3} \Gamma(1 / 3)}, b=\frac{2^{1 / 3}}{3^{2 / 3} \Gamma(2 / 3)} .
$$

The simplified form of the map can be written as

$$
\begin{aligned}
h_{k+1}= & h_{k}-F \Omega K\left(h_{k+1}\right) \sin \left(\varphi_{k}+\frac{\pi \Omega}{\left|h_{k}\right|^{3 / 2}}+\chi\right), \\
\varphi_{k+1}= & \varphi_{k}+\frac{\pi \Omega}{\left|h_{k}\right|^{3 / 2}}+\frac{\pi \Omega}{\left|h_{k+1}\right|^{3 / 2}}-F \Omega \frac{d K\left(h_{k+1}\right)}{d h_{k+1}} \\
& \times \cos \left(\varphi_{k}+\frac{\pi \Omega}{\left|h_{k}\right|^{3 / 2}}+\chi\right) .
\end{aligned}
$$

The corresponding generating function is

$$
S\left(t_{k}, h_{k+1}\right)=-K\left(h_{k+1}\right) \cos \left(\Omega t_{k}+\frac{\pi \Omega}{\left|h_{k+1}\right|^{3 / 2}}+\chi\right) .
$$

The map (96) is a general form of the Kepler map written in terms of canonical variables. Both variables $\varphi_{k}=\Omega t_{k}, h_{k}$ $=H\left(t_{k}\right)$ are defined at the same section $\Sigma_{s}$ of phase space $(x, p)$ located at the maximum distance from the center $x$ $=0$ (aphelion). The Kepler map obtained im [23,27] can be recovered from Eqs. (96) shifting the time $t$ (or the phase $\varphi$ ) by the half-period of unperturbed motion, $\pi / \omega(H)$ [or $\pi \Omega / \omega(H)]$, i.e.,

$$
\varphi_{k}+\frac{\pi \Omega}{\left|h_{k}\right|^{3 / 2}}=\bar{\varphi}_{k} \rightarrow \varphi_{k} .
$$

The Kepler map in terms of noncanonical variables has been derived in Refs. [22-25] by calculating the increments of energy $H$ and phase $\varphi$ over one phase rotation in phase space. It has the following form (in our notations):

$$
\begin{gathered}
h_{k+1}=h_{k}-F \Omega K_{0} \sin \bar{\varphi}_{k}, \\
\bar{\varphi}_{k+1}=\bar{\varphi}_{k}+\frac{2 \pi \Omega}{\left|h_{k}\right|^{3 / 2}},
\end{gathered}
$$

where $K_{0}$ is the asymptotics of the integral (95) at the highfrequency limit $\nu \rightarrow \infty: K_{0}=-2 a \pi 2^{2 / 3} / \Omega^{5 / 3}$. The Kepler map in noncanonical variables (97) can be formally obtained from the general form of the symplectic mapping (42) replacing $K(H)$ by its asymptotics of $K_{0}$, putting $L(0)=0$, and shifting the phase $\varphi_{k} \rightarrow \bar{\varphi}_{k}$.

In Refs. [22-25], the Kepler map in the form (97) has been employed to study the classical chaotic ionization of hydrogen atoms in a microwave field which has been investigated experimentally (see [56] and references therein). The map obtained in [23,27], which is equivalent to the map (96), allowed us to analyze the frequency dependence of the ionization process and to study the adiabatic and chaotic regimes of ionization.

The Kepler map of type (97) was also proposed in Refs. [28-31] to study the chaotic motion of comets near parabolic orbits in the Solar System. Particularly, in Ref. [31] using this map it has been shown that the motion of Halley's comet is chaotic.

We should emphasize that the variables in the map obtained in Refs. [23,27] as well as in the Kepler map (97) are defined at different sections of the phase space: the energy $H$ is at the maximum distance from the center (aphelion), and the phase $\bar{\varphi}$ (or time $t$ ) at the minimum distance (perihelion). Because of this, the variables $H$ and $\varphi$ are not canonically conjugated. The Kepler map in canonical variables has also been constructed in Refs. [48,49] by integrating Hamiltonian equations in extended phase space. However, the map obtained in such a way has a complicated form, although it is in good agreement with direct numerical integrations.

\section{CONCLUSIONS}

In summary, we have developed a systematic and rigorous method to construct canonical mappings near the separatrix of generic Hamiltonian systems subjected to time-dependent perturbations. The method is based on canonical transformations of variables in Hamiltonian equations of motion, unlike a conventional method to derive separatrix mappings via calculations of increments of energy and time. An important advantage of this method is that it constructs mappings in canonical variables while the conventional method yields separatrix mappings in terms of noncanonical variables, namely, energy and time defined at the different sections of phase space. This feature of conventional separatrix mapping makes it difficult to make a direct comparison of the original Hamiltonian system with the mapping, and therefore, in general, this separatrix mapping may not describe some specific properties of the system.

Canonical separatrix mappings with variables $(t, h)$ defined in the neighborhood of saddle points are important to 
study the dynamics and statistical properties of chaotic motion in a stochastic layer formed near separatrices of the system. This is because of the fact that trajectories spend most of the time near saddle points, and therefore the whole dynamics is mainly determined by the phase-space structure of the system in the neighborhood of saddle points (see Ref. [46] and references therein).

The method of canonical transformations for constructing mappings near separatrices also sheds light on attempts made in several works (see Refs. [19,32,38]) to construct a socalled exact separatrix mapping for large perturbation parameter $\epsilon$. Although one can formally write down exact mapping, similar to Eqs. (16), it is impossible to find an exact solution for the generating function. Usually one retains only the generating function in the first order of perturbation parameter $\epsilon$. In this sense, the attempt (see Refs. [19,38]) to obtain the exact map from the conventional separatrix mapping (79) by replacing the asymptotics of frequency of motion $\omega(H)$ by its exact value, while keeping the same Melnikov integrals, is not correct.

\section{ACKNOWLEDGMENTS}

The author gratefully acknowledges discussions with Professor G. Eilenberger. This work has been partially supported by the project SFB 591 of Deutsche Forschungsgemeinschaft (DFG).

\section{APPENDIX A: ASYMPTOTIC ESTIMATIONS OF THE INTEGRAL $K(h)$}

We write the integrals (87) as $K(h)+i L(h)=R(h)$,

$$
R(\eta)=2 a \int_{0}^{\pi} f(x) e^{i \eta x} d x, f(x)=\frac{\sin x}{1-a \cos x},
$$

where $a=\sqrt{1-|h|}, \eta=\lambda /|h|^{1 / 2}$. For the integer values of $\eta$ $=n$, the integral (A1) may be reduced to an integral of the type

$$
\int_{0}^{\pi} \frac{\cos [(\eta \pm 1) x]}{1-a \cos x} d x=\frac{\pi}{\sqrt{1-a^{2}}}\left(\frac{1-\sqrt{1-a^{2}}}{a}\right)^{\eta \pm 1} .
$$

Then the integral (A1) can be presented as a sum,

$$
R(\eta)=2 \pi\left(\frac{1-\sqrt{1-a^{2}}}{a}\right)^{\eta}+R_{\mathrm{osc}}(\eta)
$$

where $R_{\mathrm{osc}}(\eta)$ is the oscillatory corrections to the integral due to the noninteger values of $\eta$. In order to find these corrections, consider large values of $\eta \gg 1$. Then the oscillatory correction can be found by asymptotic expansion of the integral in a series of power of $\eta^{-1}$. We will find the asymptotic expansion by integration by part.

Integrating Eq. (A1) by part $N$ times, one can obtain

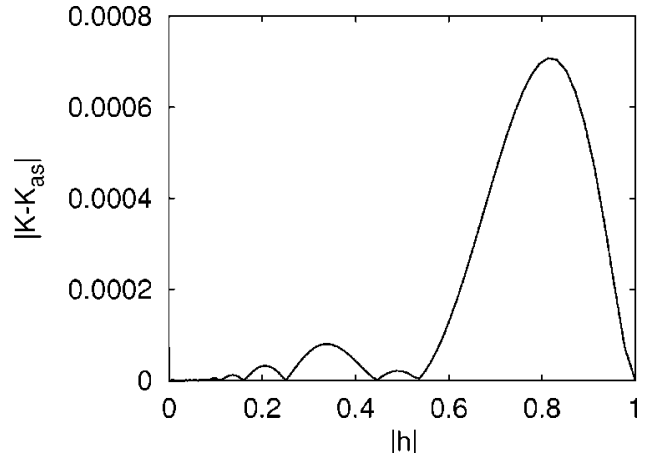

FIG. 15. Deviation of the asymptotic formula (A4) for $K(h)$ from the numerically integrated one. Parameter $\lambda=2$.

$$
\int_{0}^{\pi} f(x) e^{i \eta x} d x=\left.e^{i \eta \pi} \sum_{k=1}^{N} \frac{1}{(i \eta)^{k}} f^{(k-1)}(x)\right|_{x=\pi}+O\left(\eta^{-N-1}\right),
$$

where $f^{(k)}(x)$ is the $k$ th derivative of the function $f(x)$. One can show that $f^{(2 s)}(0)=f^{(2 s)}(\pi)=0 \quad(s=0,1,2, \ldots)$. For the odd $k=2 s+1$, the derivatives $f^{(2 k+1)}(0)$ have nonzero values. For the first two nonzero derivatives, we obtain

$$
\begin{aligned}
& a_{1} \equiv f^{(1)}(0)=\frac{1}{1-a}, \quad a_{2} \equiv f^{(3)}(0)=-\frac{1+a+2 a^{2}}{(1-a)^{3}}, \\
& b_{1} \equiv f^{(1)}(\pi)=-\frac{1}{1+a}, \quad b_{2} \equiv f^{(3)}(\pi)=\frac{1-a-2 a^{2}}{(1+a)^{3}} .
\end{aligned}
$$

Therefore, the expansion of $f(h)$ in a series of power of $1 / \eta$ up to fourth terms is given by

$$
K_{\mathrm{osc}}(\eta)=2 a \eta^{-2}\left(b_{1}-b_{2} \eta^{-2}\right) \sin (\pi \eta)+O\left(\eta^{-6}\right) .
$$

Putting $a=\sqrt{1-|h|}$ and $\eta=\lambda /|h|^{1 / 2}$, we have obtained the following asymptotic formula for $w(h)$ :

$$
\begin{aligned}
K_{\mathrm{as}}(h)= & 2 \pi\left(\frac{1-|h|^{1 / 2}}{\sqrt{1-|h|}}\right)^{\lambda /|h|^{1 / 2}} \\
& +\frac{2|h| \sqrt{1-|h|}}{\lambda^{2}} \sin \left(\frac{\pi \lambda}{|h|^{1 / 2}}\right)\left(b_{1}-b_{3} \frac{|h|}{\lambda^{2}}\right)+O\left(\frac{|h|^{3}}{\lambda^{6}}\right) .
\end{aligned}
$$

At the limit $|h| \rightarrow 0$, we have

$$
K(h) \rightarrow K=2 \pi \exp (-\lambda) .
$$

The asymptotics of the integral $K(h)$ given by Eq. (A4) is plotted in Fig. 13 at a fixed value of the parameter $\lambda=2$. The asymptotic formula (A4) is in sufficiently good agreement with the values of $K(h)$ obtained by numerical integration of the integral (A1). The deviation of Eq. (A4) from the numerical $K(h)$ is shown in Fig. 15. The maximal deviation $\max \left|K(h)-K_{\mathrm{as}}(h)\right|$ is less than $8 \times 10^{-4}$. 
[1] H. Poincaré, Les Méthodes de la Mécanique Céleste (GauthierVillars, Paris, 1892), Vols. 1-3 [English translation: New Methods of Celestial Mechanics, (AIP, New York, 1992), Vols. $1-3]$.

[2] V.K. Melnikov, Trans. Mosc. Math. Soc. 12, 1 (1963).

[3] B.V. Chirikov, Phys. Rep. 52, 265 (1979).

[4] B.V. Chirikov Nonlinear Resonance (Novosibirsk State University, Novosibirsk, 1977).

[5] G.M. Zaslavsky, R.Z. Sagdeev, D.A. Usikov, and A.A. Chernikov, Weak Chaos and Quasi-regular Patterns (Cambridge University Press, Cambridge, 1991).

[6] J.B. Weiss and E. Knobloch, Phys. Rev. A 40, 2579 (1989).

[7] T. Ahn and S. Kim, Phys. Rev. E 49, 2900 (1994).

[8] M. Latka and B.J. West, Phys. Rev. E 52, 3252 (1995).

[9] E. Weeks, J.S. Urbach, and H. Swinney, Physica D 97, 291 (1996).

[10] T. Ahn and S. Kim, Int. J. Bifurcation Chaos Appl. Sci. Eng. 7, 1025 (1997).

[11] D. del Castillo-Negrete, Phys. Fluids 10, 576 (1998).

[12] L. Kuznetsov and G.M. Zaslavsky, Phys. Rev. E 58, 7330 (1998).

[13] T. Yamagishi, Fusion Technol. 27, 505 (1995).

[14] S.S. Abdullaev and G.M. Zaslavsky, Phys. Plasmas 2, 4533 (1995).

[15] S.S. Abdullaev and G.M. Zaslavsky, Phys. Plasmas 3, 516 (1996).

[16] S.S. Abdullaev and K.H. Finken, Nucl. Fusion 38, 531 (1998).

[17] D.F. Escande, in Proceedings of the International Workshop on Plasma Theory and Nonlinear and Turbulent Processes in Physics, Kiev, 1987, edited by V.G. Bar'yakhtar, V.M. Chernousenko, N.S. Erokhin, A.G. Sitenko, and A.V. Zakharov (World Scientific, Singapore, 1988), pp. 398-430.

[18] A.J. Lichtenberg and B. Wood, Phys. Rev. A 39, 2153 (1989).

[19] I.I. Shevchenko, Phys. Scr. 57, 185 (1998).

[20] I.I. Shevchenko, Celest. Mech. Dyn. Astron. 73, 259 (1999).

[21] I.I. Shevchenko and V.V. Kouprianov, Astron. Astrophys. 394, 663 (2002)

[22] G. Casati, I. Cuarneri, and D.L. Shepelyansky, Phys. Rev. A 36, 3501 (1987).

[23] V. Gontis and B. Kaulakys, J. Phys. B 20, 5051 (1987).

[24] G. Casati, I. Cuarneri, and D.L. Shepelyansky, IEEE J. Quantum Electron. 24, 1420 (1988).

[25] R.V. Jensen, J.G. Leopold, and D. Richards, J. Phys. B 21, L527 (1988).

[26] G. Casati, I. Cuarneri, and D.L. Shepelyansky, Physica A $\mathbf{1 6 3}$, 205 (1990).

[27] B. Kaulakys and G. Vilutis, Phys. Scr. 59, 251 (1999).

[28] T.Y. Petrosky, Phys. Lett. A 117, 328 (1986).
[29] R.Z. Sagdeev and G.M. Zaslavsky, Nuovo Cimento Soc. Ital. Fis., B 97, 119 (1987).

[30] T.Y. Petrosky and R. Broucke, Celest. Mech. 42, 53 (1988).

[31] B.V. Chirikov and V.V. Vecheslavov, Astron. Astrophys. 221, 146 (1989).

[32] T. Ahn, S. Kim, and G. Kim, Physica D 89, 315 (1996).

[33] S.S. Abdullaev, J. Phys. A 32, 2745 (1999).

[34] D. Treschev, J. Nonlinear Sci. 12, 27 (2002).

[35] V.V. Vecheslavov, JETP Lett. 63, 1047 (1996).

[36] V.V. Vecheslavov, Physica D 131, 55 (1999).

[37] A.C.J. Luo and R.P.S. Han, Chaos, Solitons Fractals 11, 2349 (2000).

[38] I.I. Shevchenko, J. Exp. Theor. Phys. 91, 615 (2000).

[39] A.C.J. Luo and R.P.S. Han, Chaos, Solitons Fractals 12, 2493 (2001).

[40] A.C.J. Luo, Int. J. Bifurcation Chaos Appl. Sci. Eng. 12, 409 (2002).

[41] S.S. Abdullaev and G.M. Zaslavsky, Bull. Am. Phys. Soc. 39, 1659 (1994).

[42] G.M. Zaslavsky and S.S. Abdullaev, Phys. Rev. E 51, 3901 (1995).

[43] S.S. Abdullaev, Phys. Lett. A 234, 281 (1997).

[44] L. Kuznetsov and G.M. Zaslavsky, Phys. Rep. 288, 457 (1997).

[45] S.S. Abdullaev and K.H. Spatschek, Phys. Rev. E 60, R6287 (1999).

[46] S.S. Abdullaev, Phys. Rev. E 62, 3508 (2000).

[47] L. Kuznetsov and G.M. Zaslavsky, Phys. Rev. E 66, 046212 (2002).

[48] M. Nauenberg, Europhys. Lett. 13, 611 (1990).

[49] P. Pakoński and J. Zakrzewski, Acta Phys. Pol. B 32, 2801 (2001).

[50] S.S. Abdullaev, J. Phys. A 35, 2811 (2002).

[51] V.V. Vecheslavov, J. Exp. Theor. Phys. 92, 744 (2001).

[52] V.V. Vecheslavov and B.V. Chirikov, J. Exp. Theor. Phys. 93, 649 (2001).

[53] N.N. Filonenko and G.M. Zaslavsky, Sov. Phys. JETP 27, 851 (1968).

[54] M.J. Davis and R.E. Wyatt, Chem. Phys. Lett. 86, 235 (1982).

[55] M.E. Goggin and P.W. Milonni, Phys. Rev. A 37, 796 (1988).

[56] R.V. Jensen, M.M. Susskind, and M.M. Sanders, Phys. Rep. 201, 1 (1991).

[57] Handbook on Mathematical Functions, edited by M. Abramowitz and I. Stegun (Dover, New York, 1965).

[58] R. McLachlan and P. Atela, Nonlinearity 5, 541 (1992).

[59] As was recently shown in Refs. [51,52], in some class of Hamiltonian systems separatrices are not destroyed even for moderately large perturbations. 